\title{
Generating Posets beyond N
}

\author{
Uli Fahrenberg ${ }^{1}$, Christian Johansen ${ }^{2}$, Georg Struth ${ }^{3}$, Ratan Badahur Thapa ${ }^{2}$ \\ 1 École Polytechnique, Palaiseau, France \\ 2 University of Oslo, Norway \\ 3 University of Sheffield, UK
}

\begin{abstract}
We introduce iposets - posets with interfaces - equipped with a novel gluing composition along interfaces and the standard parallel composition. We study their basic algebraic properties as well as the hierarchy of gluing-parallel posets generated from singletons by finitary applications of the two compositions. We show that not only series-parallel posets, but also interval orders, which seem more interesting for modelling concurrent and distributed systems, can be generated, but not all posets. Generating posets is also important for constructing free algebras for concurrent semirings and Kleene algebras that allow compositional reasoning about such systems.
\end{abstract}

\section{Introduction}

This work is inspired by Tony Hoare's programme of building graph models of concurrent Kleene algebra (CKA) 12 for real-world applications. CKA extends the sequential compositions, nondeterministic choices and unbounded finite iterations of imperative programs modelled by Kleene algebra into concurrency, adding operations of parallel composition and iteration, and a weak interchange law for the sequential-parallel interaction. Such algebras have a long history in concurrency theory, dating back at least to Winkowski 35. Commutative Kleene algebra - the parallel part of CKA - has been investigated by Pilling and Conway [2]. A double semiring with weak interchange - CKA without iteration-has been introduced by Gischer [8; ; its free algebras have been studied by Bloom and Ésik [1]. CKA, like Gischer's concurrent semiring, has both interleaving and true concurrency models, e.g. shuffle as well as pomset languages. Series-parallel pomset languages, which are generated from singletons by finitary applications of sequential and parallel compositions, form free algebras in this class [19, 22 (at least when parallel iteration is ignored). The inherent compositionality of algebra is thus balanced by the generative properties of this model. Yet despite this and other theoretical work, applications of CKA remain rare.

One reason is that series-parallel pomsets are not expressive enough for many real-world applications: even simple producer-consumer examples cannot be modelled [24. Tests, which are needed for the control structure of concurrent programs and as assertions, are hard to capture in models of CKA (see 17 and its discussion in [18). Finally, it remains unclear how modal operators could be 
defined over graph models akin to pomset languages, which is desirable for concurrent dynamic algebras and logics beyond alternating nondeterminism [7,28.

A natural approach to generating more expressive pomset languages is to "cut across" pomsets in more general ways when (de)composing them. This can be achieved by (de)composing along interfaces, and this idea can be traced back again to Winkowski [35]; see also [3,4,25] for interface-based compositions of graphs and posets, or [13, 26, 27, for recent interface-based graph models for CKA. As a side effect, interfaces may yield notions of tests or modalities. When they consist of events, cutting across them presumes that they extend in time and thus form intervals. Interval orders [5, 34 of events with duration have been applied widely in partial order semantics of concurrent and distributed systems [15, 20, 21, 30 33] and the verification of weak memory models [11, yet generating them remains an open problem [16].

Our main contribution lies in a new class and algebra of posets with interfaces (iposets) based on these ideas. We introduce a new gluing composition that acts like standard serial po(m)set composition outside of interfaces, yet glues together interface events, thus composing events that did not end in one component with those that did not start in the other one. Our definitions are categorical so that isomorphism classes of posets are considered ab initio. Their decoration with labels is then trivial, so that we may focus on posets instead of pomsets.

Our main technical results concern the hierarchy of gluing-parallel posets generated by finitary applications of this gluing composition and the standard parallel composition of po(m)sets, starting from singleton iposets 4 It is obvious that all series-parallel pomsets can be generated, but also all interval orders are captured at the second alternation level of the hierarchy. Beyond that, we show that the gluing-parallel hierarchy does not collapse and that posets with certain zigzag-shaped induced subposets are excluded. Yet a precise characterisation of the generated (i)posets remains open. Series-parallel posets, by comparison, exclude precisely those posets with induced $\mathrm{N}$-shaped subposets; interval orders exclude precisely those with induced subposets $2+2$, which makes the two classes incomparable. Iposets thus retain at least the pleasant compositionality properties of series-parallel pomsets and the wide applicability of interval orders in concurrency and distributed computing.

In addition, we establish a bijection between isomorphism classes of interval orders and certain equivalence classes of interval sequences [30], and we study the basic algebraic properties of iposets, including weak interchange laws and a Levi lemma. The relationship between gluing-parallel ipo(m)set languages and CKA is left for another article.

\section{Posets and Series-Parallel Posets}

A poset $(P, \leq)$ is a set $P$ equipped with a partial order $\leq$; a reflexive, transitive, antisymmetric relation $\leq$ on $P$. A morphism of posets $P$ and $Q$ is an order-

\footnotetext{
${ }^{4}$ There is only one singleton poset, but with interfaces, there are four singleton iposets.
} 
preserving function $f: P \rightarrow Q$, that is, $x \leq_{P} y$ implies $f(x) \leq_{Q} f(y)$. Posets and their morphisms define the category Pos.

A poset is linear if each pair of elements is comparable with respect to its order. We write $<$ for the strict part of $\leq$. We write $[n]$, for $n \geq 1$, for the discrete $n$-poset $(\{1, \ldots, n\}, \leq)$, which satisfies $i \leq j \Leftrightarrow i=j$. Additionally, $[0]=\emptyset$.

The isomorphisms in Pos are order bijections: bijective functions $f: P \rightarrow Q$ for which $x \leq_{P} y \Leftrightarrow f(x) \leq_{Q} f(y)$. We write $P \cong Q$ if posets $P$ and $Q$ are isomorphic. We generally consider posets up-to isomorphism and assume, moreover, that all posets are finite.

Concurrency theory often considers (isomorphism classes of) posets with points labelled by letters from some alphabet, which represent actions of some concurrent system. These are known as partial words or pomsets. As we are mainly interested in structural aspects of concurrency, we ignore such labels.

Series-parallel posets form a well investigated class that can be generated from the singleton poset by finitary applications of two compositions. Their labelled variants generalise rational languages into concurrency. For arbitrary posets, these compositions are defined as follows.

Definition 1. Let $P_{1}=\left(P_{1}, \leq_{1}\right)$ and $P_{2}=\left(P_{2}, \leq_{2}\right)$ be posets.

1. Their serial composition is the poset $P ; Q=\left(P \sqcup Q, \leq_{1} \cup \leq_{2} \cup P_{1} \times P_{2}\right)$.

2. Their parallel composition is the poset $P_{1} \otimes P_{2}=\left(P_{1} \sqcup P_{2}, \leq_{1} \cup \leq_{2}\right)$.

Here, $\sqcup$ means disjoint union (coproduct) of sets. We generalise serial composition to a gluing composition in Section 4, after equipping posets with interfaces.

Serial and parallel compositions respect isomorphism, and $[n+m]$ is isomorphic to $[n] \otimes[m]$ with isomorphism $\varphi_{n, m}:[n+m] \rightarrow[n] \otimes[m]$ given by

$$
\varphi_{n, m}(i)= \begin{cases}i_{[n]} & \text { if } i \leq n \\ (i-n)_{[m]} & \text { if } i>n\end{cases}
$$

By definition, a poset is series-parallel (an sp-poset) if it is either empty or can be obtained from the singleton poset by applying the serial and parallel compositions a finite number of times. It is well known [10,29] that a poset is series-parallel iff it does not contain the induced subposet $\mathrm{N}=(\cdot \vec{\longrightarrow} \cdot)$ 5

Sp-po(m)sets form bi-monoids with respect to serial and parallel composition, and with the empty poset as shared unit - in fact the free algebras in this class. Compositionality of the recursive definition of sp-po(m)sets is thus reflected by the compositionality of their algebraic properties, which is often considered a desirable property of concurrent systems 33. Yet sp-posets are, in fact, too compositional for many applications: even simple consumer-producer problems inevitably generate N's 24], as shown in Fig. 1] which contains the N spanned by $c_{1}, c_{2}, p_{2}$, and $p_{3}$ as an induced subposet among others.

\footnotetext{
${ }^{5}$ This means that there is no injection $f$ from $\mathrm{N}$ satisfying $x \leq y \Leftrightarrow f(x) \leq f(y)$.
} 


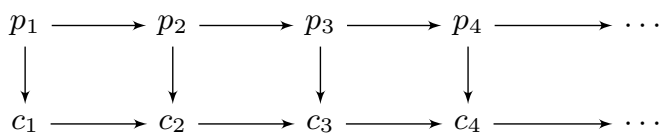

Fig. 1. The producer-consumer example

\section{Interval orders and interval sequences}

Interval orders 5, 34 form another class of posets that are ubiquitous in concurrent and distributed computing. Intuitively, they are isomorphic to sets of intervals on the real line that are ordered whenever they do not overlap.

Definition 2. An interval order is a relational structure $(P,<)$ with $<$ irreflexive such that $w<y$ and $x<z$ imply $w<z$ or $x<y$, for all $w, x, y, z \in P$.

Transitivity of $<$ follows. An alternative geometric characterisation is that interval orders are precisely those posets that do not contain the induced subposet $2+2=(\cdot \vec{\longrightarrow} \cdot)$.

The intuition is captured by Fishburn's theorem [5], which implies that a finite poset $P$ is an interval order iff it has an interval representation: a pair of functions $b, e: P \rightarrow Q$ into some linear order $\left(Q,<_{Q}\right)$ such that $b(x)<_{Q} e(x)$, for all $x \in P$, and $x<_{P} y \Leftrightarrow e(x)<_{Q} b(y)$, for all $x, y \in P$. By the first condition, pairs $(b(x), e(x))$ correspond to intervals $I(x)=[b(x), e(x)]$ in $Q$; by the second condition, $x<_{P} y$ iff $I(x)$ lies entirely before $I(y)$ in $Q$.

We write $\rho_{I}(P)$ for the set of interval representations of $P$. Each representation can be rearranged such that all endpoints of intervals are distinct ([9], Lemma 1.5). We henceforth assume that all interval presentations have this property. It then holds that $|Q|=2|P|$, and we can fix $Q$ as the target type of any interval representation of $P$.

Finally, with relation $\sqsubset$ on the set of maximal antichains of poset $P$ given by

$$
A \sqsubset B \Leftrightarrow(\forall x \in A \backslash B . \forall y \in B \backslash A . x<y),
$$

it has been shown that $P$ is an interval order iff $\sqsubset$ is a strict linear order 6 .

Interval orders also occur implicitly in the ST-traces of Petri nets 30. In a pure order-theoretic setting, these are interval sequences, that is, sequences of $b(x)$ and $e(x)$, with $x$ from some finite set $P$, in which each $b(x)$ occurs exactly once and each $e(x)$ at most once and only after the corresponding $b(x)$. An interval sequence is closed if each $e(x)$ occurs exactly once 30,33. An interval trace [16] is an equivalence class of interval sequences modulo the relations $b(x) b(y) \approx b(y) b(x)$ and $e(x) e(y) \approx e(y) e(x)$ for all $x, y \in P$. We write $\approx^{*}$ for the congruence generated by $\approx$ on interval sequences. We identify interval sequences and interval traces with the Hasse diagrams of their linear orders over $Q$.

Lemma 3. Let $P$ be an interval order and $(b, e) \in \rho_{I}(P)$. Then $\left(Q,<_{Q}\right)$ is a closed interval sequence. 
Proof. Trivial.

We write $\sigma_{(b, e)}(P)$ for the interval sequence of interval order $P$ and $(b, e) \in \rho_{I}(P)$, and $\Sigma(P)$ for the set of all interval sequences of interval representations of $P$.

Lemma 4. If $\sigma \in \Sigma(P)$ and $\sigma \approx^{*} \sigma^{\prime}$, then $\sigma^{\prime} \in \Sigma(P)$.

Proof (sketch). We show that $\sigma \in \Sigma(P)$ and $\sigma \approx \sigma^{\prime}$ imply $\sigma^{\prime} \in \Sigma(P)$. Suppose that $\sigma=\sigma_{1} b(x) b(y) \sigma_{2}$ and $\sigma^{\prime}=\sigma_{1} b(y) b(x) \sigma_{2}$ and that $(b, e) \in \rho_{I}(P)$ generates $\sigma$. Then $\left(b^{\prime}, e\right)$ with

$$
b^{\prime}(z)= \begin{cases}b(y), & \text { if } z=x \\ b(x), & \text { if } z=y \\ b(z), & \text { otherwise }\end{cases}
$$

is in $\rho_{I}(P)$, as $b^{\prime}(x)<_{Q} e(x), b^{\prime}(y)<_{Q} e(y)$ and, for all $v, w \in P, v<_{P} w \Leftrightarrow$ $e(v)<_{P} b(w)$ still holds. In addition, $\left(b^{\prime}, e\right)$ generates $\sigma^{\prime}$. An analogous result for $\sigma=\sigma_{1} e(x) e(y) \sigma_{2}$ and $\sigma^{\prime}=\sigma_{1} e(y) e(x) \sigma_{2}$ holds by opposition. The result for $\approx^{*}$ follows by a simple induction.

Lemma 5. Let $P$ be an interval order. If $(b, e),\left(b^{\prime}, e^{\prime}\right) \in \rho_{I}(P)$ assign $b$ and $e$ to elements of $P$ in interval sequences, then $\sigma_{(b, e)}(P) \approx^{*} \sigma_{\left(b^{\prime}, e^{\prime}\right)}(P)$.

Proof (sketch). Let $\prec_{1}$ and $\prec_{2}$ be the orderings of the interval sequences for $(b, e)$ and $\left(b^{\prime}, e^{\prime}\right)$ in $Q$. Then $b(x) \prec_{1} e(x)$ and $b(x) \prec_{2} e(x)$ for all $x \in X$, and $e(x) \prec_{1} b(y) \Leftrightarrow e(x) \prec_{2} b(y)$ for all $x, y \in X$. It follows that there is no $b(z)$ in $\prec_{1}$ or $\prec_{2}$ between the positions of $e(x)$ in $\prec_{1}$ and $\prec_{2}$ and, by opposition, there is no $e(z)$ in $\prec_{1}$ or $\prec_{2}$ between the positions of $b(x)$ in $\prec_{1}$ and $\prec_{2}$. But this means that the positions of $e(x)$ and $b(x)$ can be rearranged by $\approx^{*}$.

Proposition 6. If $P$ is an interval order and $(b, e) \in \rho_{I}(P)$, then $\left[\sigma_{(b, e)}(P)\right]_{\approx^{*}}=$ $\Sigma(P)$. The mapping $\varphi$ defined by $\varphi(P)=\left[\sigma_{(b, e)}(P)\right]_{\approx^{*}}$ is a bijection.

Proof. By Lemma 4 and 5, and properties of interval representations.

\section{Posets with interfaces}

An element $s$ of poset $(P, \leq)$ is minimal (maximal) if $v \not \leq s(v \nsupseteq s)$ holds for all $v \in P$. We write $P_{\min }\left(P_{\max }\right)$ for the sets of minimal (maximal) elements of $P$.

Definition 7. A poset with interfaces (iposet) consists of a poset $P$ together with two injective morphisms

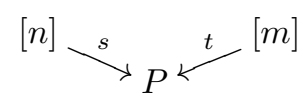

such that $s[n] \subseteq P_{\min }$ and $t[m] \subseteq P_{\max }$. 

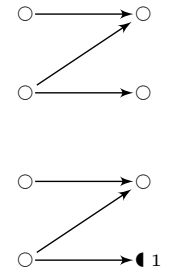
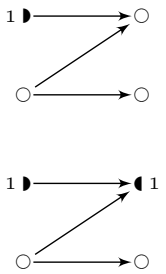
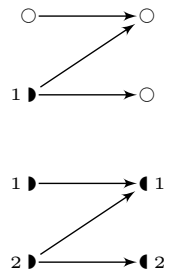
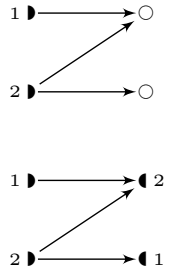

Fig. 2. Eight of 25 different iposets based on poset N.

Injection $s:[n] \rightarrow P$ represents the source interface of $P$ and $t:[m] \rightarrow P$ its target interface. We write $(s, P, t): n \rightarrow m$ for the iposet $s:[n] \rightarrow P \leftarrow[m]: t$.

Figure 2 shows some examples of iposets. Elements of source and target interfaces are depicted as filled half-circles to indicate the unfinished nature of the events they represent.

Next we define a sequential gluing composition on iposets whose interfaces agree and we adapt the standard parallel composition of posets to iposets.

Definition 8. Let $\left(s_{1}, P_{1}, t_{1}\right): n \rightarrow m$ and $\left(s_{2}, P_{2}, t_{2}\right): \ell \rightarrow k$ be iposets.

1. For $m=\ell$, their gluing composition is the iposet $\left(s_{1}, P_{1} \triangleright P_{2}, t_{2}\right): n \rightarrow k$ with $P_{1} \triangleright P_{2}=\left(\left(P_{1} \sqcup P_{2}\right) / t_{1}(i)=s_{2}(i), \leq_{1} \cup \leq_{2} \cup\left(P_{1} \backslash t_{1}[m]\right) \times\left(P_{2} \backslash s_{2}[m]\right)\right)$.

2. Their parallel composition is the iposet $\left(s, P_{1} \otimes P_{2}, t\right): n+\ell \rightarrow m+k$ with $s=\left(s_{1} \otimes s_{2}\right) \circ \varphi_{n, l}$ and $t=\left(t_{1} \otimes t_{2}\right) \circ \varphi_{m, k}$.

Parallel composition of iposets thus puts components "side by side": it is the disjoint union of posets and interfaces. Gluing composition puts iposets "one after the other", $P_{1}$ before $P_{2}$, but glues their interfaces together (and adds arrows from all points in $P_{1}$ that are not in its target interface to all points in $P_{2}$ that are not in its source interface). Figures 3 and 4 show examples. The half-circles in source and target interfaces are glued to circles in the diagrams.

We define identity iposets $\mathrm{id}_{n}=$ (id, $[n]$, id) $: n \rightarrow n$, for $n \geq 0$. For convenience, we generalise this notation to other singleton posets with interfaces: for $k, \ell \leq n$, we write ${ }^{k}$ id $_{n}^{\ell}$ for the iposet $\left(f_{k}^{n},[n], f_{\ell}^{n}\right): k \rightarrow \ell$, where $f_{k}^{n}:[k] \rightarrow[n]$ is the (identity) injection $x \mapsto x$ (similarly for $f_{\ell}^{n}$ ). Hence $\mathrm{id}_{n}={ }^{n} \mathrm{id}_{n}^{n}$. We write $\mathcal{S}=\left\{{ }^{k} \mathrm{id}_{1}^{\ell} \mid k, \ell=0,1\right\}$ for the set of all singleton iposets.

Parallel composition need not be commutative, as the namings of interfaces in $P \otimes Q$ may differ from those in $Q \otimes P$. One can, however, rename interfaces using symmetries: iposets $(s,[n], t): n \rightarrow n$ with $s$ and $t$ bijective. Figure [5 shows two parallel compositions where renaming of interfaces and gluing with another iposet yields non-isomorphic posets.

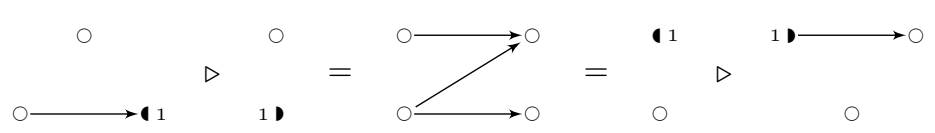

Fig. 3. Two different decompositions of the N. 

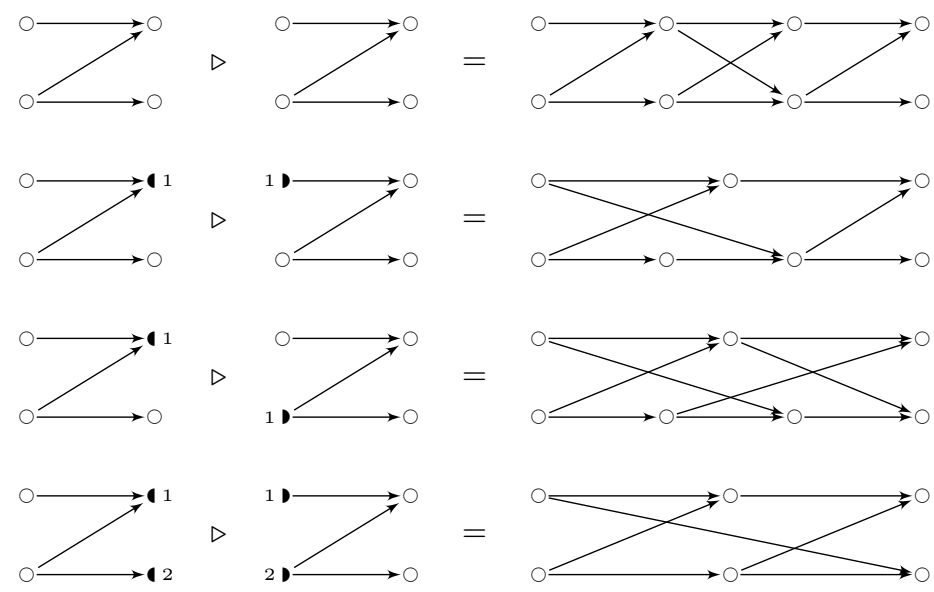

Fig. 4. Four gluings of different Ns with interfaces.

Also, gluing and parallel composition need not satisfy an interchange law:

$\left({ }^{0} \mathrm{id}_{1}^{0} \otimes{ }^{0} \mathrm{id}_{1}^{0}\right) \triangleright\left({ }^{0} \mathrm{id}_{1}^{0} \otimes{ }^{0} \mathrm{id}_{1}^{0}\right)=\left({ }^{\cdot \overrightarrow{\mathbf{S}^{*}}} \cdot\right) \neq(\cdot \vec{\longrightarrow} \cdot \vec{\cdot} \cdot)=\left({ }^{0} \mathrm{id}_{1}^{0} \triangleright^{0} \mathrm{id}_{1}^{0}\right) \otimes\left({ }^{0} \mathrm{id}_{1}^{0} \triangleright^{0} \mathrm{id}_{1}^{0}\right)$.

Hence iposets do not form (strict) monoidal categories, or even PROPs, because $\otimes$ is not a tensor. The situation differs from gluing compositions where interfaces of iposets are defined by all minimal and maximal elements [35, and also from sequential compositions of digraphs with "partial" interfaces similar to ours where interface points glue arrows together and disappear in these compositions [4]. Both of these give rise to a PROP.

Gluing composition, of course, is not commutative either:

$$
{ }^{0} \mathrm{id}_{1}^{1} \triangleright{ }^{1} \mathrm{id}_{1}^{0}={ }^{0} \mathrm{id}_{1}^{0}=(\cdot) \neq(\cdot \longrightarrow \cdot)={ }^{1} \mathrm{id}_{1}^{0} \triangleright{ }^{0} \mathrm{id}_{1}^{1}
$$

Proposition 9. Iposets form a small category with natural numbers as objects, iposets $(s, P, t): n \rightarrow m$ as morphisms, $\triangleright$ as composition, and identities $\mathrm{id}_{n}$.

Checking associativity of $\triangleright$ and the existence of units is routine, as is the proof of the next proposition.

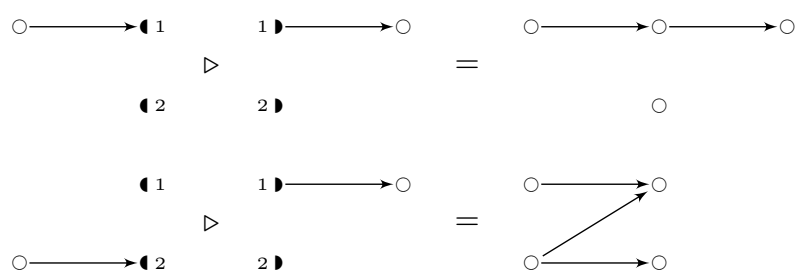

Fig. 5. Non-isomorphic gluings of symmetric parallel compositions. 
Proposition 10. Iposets form a monoid with composition $\otimes$ and unit $\mathrm{id}_{0}$.

A morphism of iposets is a commuting diagram

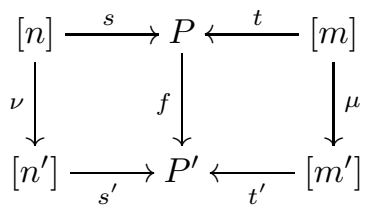

where $\nu$ and $\mu$ are strictly order preserving with respect to $<_{\mathbb{N}}$ and $f$ is an order morphism. Intuitively, iposet morphisms thus preserve interfaces and their order in $\mathbb{N}$. Let iPos denote the so-defined category.

An iposet morphism $(\nu, f, \mu)$ is an isomorphism if $\nu, f$ and $\mu$ are order isomorphisms. Hence $n=n^{\prime}, m=m^{\prime}, \nu=\mathrm{id}: n \rightarrow n$, and $\mu=\mathrm{id}: m \rightarrow m$ in diagram (11). As a consequence, we note that iposets which are related by a symmetry $(s,[n], t): n \rightarrow n$ need not be isomorphic.

We write $P \cong Q$ if there exists an isomorphism $\varphi: P \rightarrow Q$. The following lemma shows that the two compositions respect isomorphism.

Lemma 11. Let $P, P^{\prime}, Q, Q^{\prime}$ be iposets. Then $P \cong P^{\prime}$ and $Q \cong Q^{\prime}$ imply $P \otimes Q \cong$ $P^{\prime} \otimes Q^{\prime}$ and $P \triangleright Q \cong P^{\prime} \triangleright Q^{\prime}$.

Proof. Let $\varphi: P \rightarrow P^{\prime}$ and $\psi: Q \rightarrow Q^{\prime}$ be (the poset components of) isomorphisms. Define the functions $\varphi \otimes \psi: P \sqcup Q \rightarrow P^{\prime} \sqcup Q^{\prime}$ and $\varphi \triangleright \psi$ : $(P \sqcup Q)_{/ t_{P}(i)=s_{Q}(i)} \rightarrow\left(P^{\prime} \sqcup Q^{\prime}\right) / t_{P^{\prime}}(i)=s_{Q^{\prime}}(i)$ as

$$
(\varphi \square \psi)(x)= \begin{cases}\varphi(x) & \text { if } x \in P, \\ \psi(x) & \text { if } x \in Q,\end{cases}
$$

for $\square \in\{\otimes, \triangleright\}$. First, $\varphi \otimes \psi$ is obviously an isomorphism. Second, $\varphi \triangleright \psi$ is welldefined because $\varphi \circ t_{P}(i)=\psi \circ s_{Q}(i)$ for all $i \in[m]$, and easily seen to be an isomorphism as well.

We write $P \preceq Q$ if there is a bijective (on points) morphism $\varphi: Q \rightarrow P$ between iposets $P$ and $Q$. Intuitively, $P \preceq Q$ iff $P$ has more arrows and is therefore less parallel than $Q$, while interfaces are preserved. Similar relations on posets and pomsets, sometimes called subsumption, are well studied [8,10]. In particular, $\preceq$ is a preorder on (finite) iposets and a partial order up to isomorphism.

Lemma 12. For iposets $P, P^{\prime}, Q, Q^{\prime}$, the following lax interchange law holds:

$$
\left(P \otimes P^{\prime}\right) \triangleright\left(Q \otimes Q^{\prime}\right) \preceq(P \triangleright Q) \otimes\left(P^{\prime} \triangleright Q^{\prime}\right)
$$

Proof. Let $P_{\ell}=\left(P \otimes P^{\prime}\right) \triangleright\left(Q \otimes Q^{\prime}\right)$ and $P_{r}=(P \triangleright Q) \otimes\left(P^{\prime} \triangleright Q^{\prime}\right)$. First, $P_{\ell}=(P \sqcup Q)_{/ t_{P} \equiv s_{Q}} \sqcup\left(P^{\prime} \sqcup Q^{\prime}\right)_{/ t_{P^{\prime}} \equiv s_{Q^{\prime}}}=\left(P \sqcup Q \sqcup P^{\prime} \sqcup Q^{\prime}\right)_{t_{P} \equiv s_{Q}, t_{P^{\prime}} \equiv s_{Q^{\prime}}}=P_{r}$, by definition of $\otimes$. Hence both posets have the same points, and we may choose 
$\varphi: P_{r} \rightarrow P_{\ell}$ to be the identity. It remains to show that $\varphi$ is order preserving, which means that every arrow in $P_{r}$ must be in $P_{\ell}$.

Hence suppose $x \leq_{P_{r}} y$, that is, $x \leq_{P \triangleright Q} y$ or $x \leq_{P^{\prime} \triangleright Q^{\prime}} y$. In the first case, if $x \leq_{P} y$ or $x \leq_{Q} y$, then $x \leq_{P \otimes P^{\prime}} y$ or $x \leq_{Q \otimes Q^{\prime}} y$ and therefore $x \leq_{P_{\ell}} y$; and if $x \in P \backslash t_{P}$ and $y \in Q \backslash s_{Q}$, then $x \in P \sqcup P^{\prime} \backslash t_{P \otimes P^{\prime}}$ and $y \in Q \sqcup Q^{\prime} \backslash s_{Q \otimes Q^{\prime}}$ and therefore $x \leq_{P_{\ell}} y$, too. The second case is symmetric. Thus, in any case, $x \leq_{P_{\ell}} y$.

In sum, the algebra of iposets is thus similar to concurrent monoids [12, but $\triangleright$ is a partial operation with many units $i_{k}$. As $\otimes$ is not a tensor, the categorical structure of iposets is somewhat unusual and deserves further exploration.

Proposition 13. Pos embeds into iPos as iposets with both interfaces [0], and likewise for morphisms. The so-defined inclusion functor $J$ : Pos $\rightarrow$ iPos is fully faithful and left adjoint to the forgetful functor $F:$ iPos $\rightarrow$ Pos that maps $(s, P, t)$ to $P$, hence Pos is coreflective in iPos. Under F, gluing composition of iposets becomes serial composition of posets, and parallel composition of iposets becomes that of posets (hence, commutative).

Proof. It is clear that $J$ is a functor. It is full because any morphism $\tilde{f}$ from $P$ : $0 \rightarrow 0$ to $Q: 0 \rightarrow 0$ in iPos must have the form $(\emptyset, f, \emptyset)=J f$ for some $f$ in Pos. It is faithful because $J f=(\emptyset, f, \emptyset)=(\emptyset, g, \emptyset)=J g$ implies $f=g$. For $P \in$ Pos and $\tilde{Q} \in \mathrm{iPos}, J$ induces a natural bijection $J: \operatorname{Pos}(P, F \tilde{Q}) \cong \operatorname{iPos}(J P, \tilde{Q})$, hence $J$ and $F$ are indeed adjoint. The last claims about the operations are clear.

\section{$5 \quad$ Further Properties of Iposets}

We now derive additional algebraic properties of iposets, before turning to the set of iposets generated by gluing and parallel composition from singleton iposets.

For an iposet $P$ with order relation $\leq$ we write $\|=\not \leq \cap \nsucceq$. Hence $x \| y$ iff $x$ and $y$ are unrelated and therefore independent.

In addition to the lax interchange in Lemma 12, we prove an equational interchange law that shows that the equational theory of iPos as given by the bimonoidal laws in Propositions 9 and 10 is not free. The lemmas further below then show that this law is the only non-trivial additional identity.

Lemma 14 (Interchange). For all iposets $P, Q$ and $k, \ell \in\{0,1\}$,

$$
\left({ }^{k} \mathrm{id}_{1}^{1} \otimes P\right) \triangleright\left({ }^{1} \mathrm{id}_{1}^{\ell} \otimes Q\right)={ }^{k} \mathrm{id}_{1}^{\ell} \otimes(P \triangleright Q) .
$$

Proof (sketch). The interface between ${ }^{k} \mathrm{id}_{1}^{1}$ and ${ }^{1} \mathrm{id}_{1}^{\ell}$ forces these iposets to be glued separately to the rest in the gluing composition $\left({ }^{k} \mathrm{id}_{1}^{1} \otimes P\right) \triangleright\left({ }^{1} \mathrm{id}_{1}^{\ell} \otimes Q\right)$.

One the one hand, it follows that singleton iposets in $\mathcal{S}$ do not interfere with compositions. On the other hand, Lemma 14 shows that decompositions need not be unique. The next lemma shows a kind of converse: if an iposet can be decomposed by $\triangleright$ and also by $\otimes$, then all but one of the components must be in $\mathcal{S}$. Henceforth, let $\mathcal{C}_{1}=\left\{P_{1} \otimes \cdots \otimes P_{n} \mid P_{1}, \ldots, P_{n} \in \mathcal{S}\right\}$ denote the set of multisets-with-interfaces, that is, iposets with discrete order. 
Lemma 15 (Decomposition). Let $P=P_{1} \otimes P_{2}=Q_{1} \triangleright Q_{2}$ such that $P_{1} \neq \mathrm{id}_{0}$, $P_{2} \neq \mathrm{id}_{0}$, and $Q_{1} \neq{ }^{k} \mathrm{id}_{n}^{n}, Q_{2} \neq{ }^{n} \mathrm{id}_{n}^{k}$ for any $k \leq n$. Then $P_{1} \in \mathcal{C}_{1}$ or $P_{2} \in \mathcal{C}_{1}$.

Proof. Suppose $P_{1} \notin \mathcal{C}_{1}$ and $P_{2} \notin \mathcal{C}_{1}$. Then $P$ contains a $2+2$ : there are $w, x \in P_{1}$ and $y, z \in P_{2}$ for which $w<_{P} x, y<_{P} z, w\left\|_{P} y, w\right\|_{P} z, x \|_{P} y$, and $x \|_{P} z$.

If $w, y \notin Q_{2}$, then $w, y \in Q_{1} \backslash t_{Q_{1}}$. As $Q_{2} \neq{ }^{n} \mathrm{id}_{n}^{k}$ for any $k \leq n$, there must be an element $v \in Q_{2} \backslash s_{Q_{2}}$. But then $w \leq_{P} v$ and $y \leq_{P} v$, which yields arrows between $w \in P_{1}$ and $y \in P_{2}$ that contradict $P=P_{1} \otimes P_{2}$. A dual argument rules out that $x, z \notin Q_{1}$.

It follows that $w \in Q_{2}$ or $y \in Q_{2}$. Assume, without loss of generality, that $w \in Q_{2}$. Then $x \in Q_{2} \backslash s_{Q_{2}}$ because $w \leq_{P_{1}} x$. Now if also $y \in Q_{2}$, then by the same argument, $z \in Q_{2} \backslash s_{Q_{2}}$. Hence $Q_{2}$ contains two different points which are not in its starting interface; and as $Q_{1} \backslash t_{Q_{1}}$ is non-empty, this again establishes a connection between $x \in P_{1}$ and $z \in P_{2}$ which cannot exist. Hence $y \notin Q_{2}$, but then $y \in Q_{1} \backslash t_{Q_{1}}$, so that $y \leq_{P} x$, which contradicts $x \|_{P} y$.

The next lemma generalises Levi's lemma for words [23].

Lemma 16 (Levi property). Let $P \square Q=U \square V$ for $\square \in\{\triangleright, \otimes\}$. Then there is an $R$ so that either $P=U \square R$ and $R \square Q=V$, or $U=P \square R$ and $R \square V=Q$.

Proof. The proof for $\otimes$ is trivial: If $P \otimes Q=U \otimes V$, then this iposet is partitioned into three components according to $P \sqcup Q$ and $U \sqcup V$. If the decomposition of $U$ and $V$ happens within $P$, then there is an $R$ such that $P=U \otimes R$ and $R \otimes Q=V$. Otherwise, if it happens within $Q$, then there exists an $R$ such that $U=P \otimes R$ and $R \otimes V$. Finally, if $P=U$ and $Q=V$, there is nothing to show. The proof for $\triangleright$ is similar, but more tedious due to gluing.

It is instructive to find the two cases in the decomposition of $\mathrm{N}$ in Figure 3

Levi's lemma is an interpolation property: every $P \square Q=U \square V$ has a common factorisation - either $U \square R \square Q$ or $P \square R \square V$. Hence sequential and gluing decompositions at top level are equal up-to associativity (and unit laws).

The three lemmas in this section are helpful for characterising the iposets generated by $\triangleright$ and $\otimes$ from singletons. This is the subject of the next section.

\section{Generating Iposets}

Recall that $\mathcal{S}$ is the set of singleton iposets. It contains the four iposets ${ }^{0} \mathrm{id}_{1}^{0}$, ${ }^{0} \mathrm{id}_{1}^{1},{ }^{1} \mathrm{id}_{1}^{0}$ and ${ }^{1} \mathrm{id}_{1}^{1}$, that is,

$$
[0] \rightarrow[1] \leftarrow[0], \quad[0] \rightarrow[1] \leftarrow[1], \quad[1] \rightarrow[1] \leftarrow[0], \quad[1] \rightarrow[1] \leftarrow[1],
$$

with mappings uniquely determined. We are interested in the sets of iposets generated from singletons using $\triangleright$ and $\otimes$. Note that strictly speaking, ${ }^{0} \mathrm{id}_{1}^{0}$ should not count as a generator, because by Lemma 14 it is equal to ${ }^{0} \mathrm{id}_{1}^{1} \triangleright{ }^{1} \mathrm{id}_{1}^{0}$.

Definition 17. The set of gluing-parallel iposets (gp-iposets) is the smallest set that contains the empty iposet $\mathrm{id}_{0}$ and the singleton iposets in $\mathcal{S}$ and is closed under gluing and parallel composition. 
Theorem 18. The gp-iposets are generated freely by $\mathcal{S}$ in the variety of algebras satisfying the equations of Propositions 9 and 10 and Lemma 14.

Proof (sketch). Suppose $\left(A, \triangleright, \otimes,\left(1_{i}\right)_{i \geq 0}\right)$ is any algebra satisfying the equations of Propositions 9 and 10 and Lemma 14 and let $\varphi: \mathcal{S} \rightarrow A$ be any function. We need to show that $\varphi$ extends to a unique iposet morphism $\hat{\varphi}$.

We can generate any $\mathrm{id}_{n}$ as a parallel composition of $\mathrm{id}_{1}$. We map $\hat{\varphi}\left(\mathrm{id}_{i}\right) \mapsto 1_{i}$ for any $i \geq 0$, and we map any other singleton $p \in \mathcal{S}$ as $\hat{\varphi}(p)=\varphi(p)$. For complex iposets we proceed by induction on the number of elements, assuming that homomorphism laws hold for iposets with $n$ elements.

If the top composition of the size $n+1$ iposet is $\triangleright$, then we use Levi's lemma to factorise with respect to $\triangleright$ and use associativity of $\triangleright$ to establish the homomorphism property of $\hat{\varphi}$. For $\otimes$ we proceed likewise. Finally, if the top composition is ambiguous, then the decomposition lemma forces the configuration in which the interchange lemma can be applied, yielding a parallel composition of the same size. Finally, this extension is unique, as it was forced by the construction.

Now we define hierarchies of iposets generated from $\mathcal{S}$. $\left(\operatorname{If~}^{0} \mathrm{id}_{1}^{0}\right.$ were removed from $\mathcal{S}$, the hierarchy would be different only for less than two alternations of $\triangleright$ and $\otimes$.)

For any $\mathcal{Q} \subseteq$ iPos and $\square \in\{\otimes, \triangleright\}$, let

$$
\mathcal{Q}^{\square}=\left\{P_{1} \square \cdots \square P_{n} \mid n \in \mathbb{N}, P_{1}, \ldots, P_{n} \in \mathcal{Q}\right\} .
$$

Then define $\mathcal{C}_{0}=\mathcal{D}_{0}=\mathcal{S}$ and, for all $n \in \mathbb{N}$,

$$
\mathcal{C}_{2 n+1}=\mathcal{C}_{2 n}^{\otimes}, \quad \mathcal{D}_{2 n+1}=\mathcal{D}_{2 n}^{\triangleright}, \quad \mathcal{C}_{2 n+2}=\mathcal{C}_{2 n+1}^{\triangleright}, \quad \mathcal{D}_{2 n+2}=\mathcal{D}_{2 n+1}^{\otimes}
$$

(this agrees with the $\mathcal{C}_{1}$ notation used earlier). Finally, let

$$
\overline{\mathcal{S}} \stackrel{\text { def }}{=} \bigcup_{n \geq 0} \mathcal{C}_{n}=\bigcup_{n \geq 0} \mathcal{D}_{n}
$$

be the set of all iposets generated from $\mathcal{S}$ by application of $\otimes$ and $\triangleright$.

Lemma 19. For all $n \in \mathbb{N}, \mathcal{C}_{n} \cup \mathcal{D}_{n} \subseteq \mathcal{C}_{n+1} \cap \mathcal{D}_{n+1}$.

Proof. We need to check the inclusions $\mathcal{C}_{n} \subseteq \mathcal{C}_{n+1}, \mathcal{D}_{n} \subseteq \mathcal{D}_{n+1}, \mathcal{C}_{n} \subseteq \mathcal{D}_{n+1}$ and $\mathcal{D}_{0} \subseteq \mathcal{C}_{1}$. The first two are trivial by construction, plus $\mathcal{C}_{n} \subseteq \mathcal{D}_{n+1}$ and $\mathcal{D}_{n} \subseteq \mathcal{C}_{n+1}$. For the third one, note that $\mathcal{C}_{0} \subseteq \mathcal{C}_{0}^{\triangleright}=\mathcal{S}^{\triangleright}=\mathcal{D}_{0}^{\triangleright}=\mathcal{D}_{1}$. Since $\mathcal{C}_{n}$ is constructed from $\mathcal{C}_{0}$ by the same alternations of $\otimes$ and $\triangleright$ as $\mathcal{D}_{n+1}$ is constructed from $\mathcal{D}_{1}$, the inclusion holds. The proof of the fourth inclusion is similar.

Theorem 20. An iposet is in $\mathcal{C}_{2}$ iff it is an interval order.

Proof. Suppose $P \triangleright Q \in \mathcal{C}_{2}$. First it is clear that all elements of $\mathcal{C}_{1}$ are interval orders, so we will be done once we can show that the gluing composition of two interval orders is an interval orders. This is precisely the proof of Lemma 15. if $P \triangleright Q$ contains a $2+2$, then so do $P$ or $Q$. Yet we also give a direct construction: 


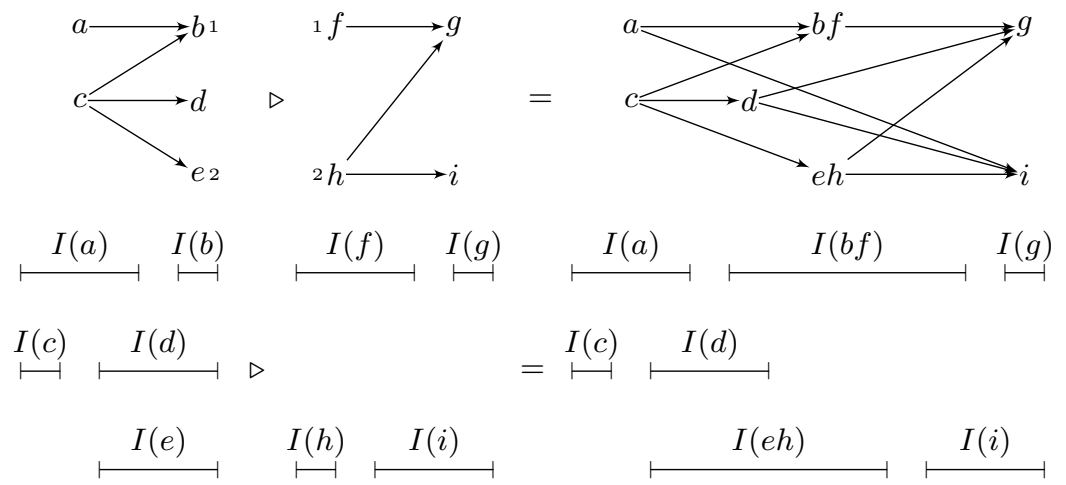

Fig. 6. Two interval orders and their concatenation: above as iposets, below using their interval representations. (Labels added for convenience.)

Let $\sigma_{P}$ be the interval sequence for interval representation $\left(b_{P}, e_{P}\right)$ of $P: n \rightarrow m$ and $\sigma_{Q}$ the interval sequence for interval representation $\left(b_{Q}, e_{Q}\right)$ of $Q: m \rightarrow k$. Then concatenate $\sigma_{P}$ and $\sigma_{Q}$, rename $b_{P}, b_{Q}$ as $b$ and $e_{P}, e_{Q}$ as $e$, delete $e\left(t_{P}(i)\right)$, $b\left(s_{Q}(i)\right)$ and replace $e\left(t_{Q}(i)\right)$ with $e\left(t_{P}(i)\right)$ for each $i \in[m]$. This yields the interval sequence for interval representation $(b, e)$ of $P \triangleright Q$ and $P \triangleright Q$ is therefore an interval order. Figure 6 gives an example.

For the backward direction, let $P$ be an interval order and $A_{P}$ its set of maximal antichains. Then $A_{P}$ is totally ordered by the relation $\sqsubset$ defined in Section 3. Now write $A_{P}=\left\{P_{1}, \ldots, P_{k}\right\}$ such that $P_{i} \sqsubset P_{j}$ for $i<j$. Then each $P_{i}$ is an element of $\mathcal{S}^{\otimes}$. Write $s_{1}:\left[n_{1}\right] \rightarrow P \leftarrow\left[n_{k+1}\right]: t_{k}$ for the sources and targets of $P$.

For $i=2, \ldots, k$, let $\left[n_{i}\right]=P_{i-1} \cap P_{i}$ be the overlap and $s_{i}:\left[n_{i}\right] \hookrightarrow P_{i}$, $t_{i-1}:\left[n_{i}\right] \hookrightarrow P_{i-1}$ the inclusions. Together with $s_{1}$ and $t_{k}$ this defines iposets $s_{i}:\left[n_{i}\right] \rightarrow P_{i} \leftarrow\left[n_{i+1}\right]: t_{i}$. (Note that $s_{1}:\left[n_{1}\right] \rightarrow P_{1}$ because $P_{1}$ is the minimal element in $A_{P}$; similarly for $t_{k}:\left[n_{k+1}\right] \rightarrow P_{k}$.) It is clear that $P=P_{1} \triangleright \cdots \triangleright P_{k}$; see also [14, Prop. 2].

In order to compare with series-parallel posets, we construct a similar hierarchy for these. Let $\mathcal{T}_{0}=\mathcal{U}_{0}=\mathcal{S}_{0}=\left\{{ }^{0} \mathrm{id}_{1}^{0}\right\}$ and, for all $n \in \mathbb{N}$,

$$
\mathcal{T}_{2 n+1}=\mathcal{T}_{2 n}^{\otimes}, \quad \mathcal{U}_{2 n+1}=\mathcal{U}_{2 n}^{\triangleright}, \quad \mathcal{T}_{2 n+2}=\mathcal{T}_{2 n+1}^{\triangleright}, \quad \mathcal{U}_{2 n+2}=\mathcal{U}_{2 n+1}^{\otimes} .
$$

Then, noting that any element of any $\mathcal{T}_{n}$ or $\mathcal{U}_{n}$ has empty interfaces and that for iposets with empty interfaces, $\triangleright$ is serial composition, we see that

$$
\overline{\mathcal{S}}_{0} \stackrel{\text { def }}{=} \bigcup_{n \geq 0} \mathcal{T}_{n}=\bigcup_{n \geq 0} \mathcal{U}_{n}
$$

is the set of series-parallel posets. Note that $\mathcal{T}_{n} \subseteq \mathcal{C}_{n}$ and $\mathcal{U}_{n} \subseteq \mathcal{D}_{n}$ for all $n$, hence also $\overline{\mathcal{S}}_{0} \subseteq \overline{\mathcal{S}}$. Now $\overline{\mathcal{S}}_{0}$ contains precisely the $\mathrm{N}$-free posets whereas $\mathrm{N}$ is an interval order. Hence $\mathrm{N} \in \mathcal{C}_{2}$, implying the next lemma. On the other hand, we will see below that $\overline{\mathcal{S}}_{0} \nsubseteq \mathcal{C}_{n}$ for any $n$. 
Lemma 21. $\mathcal{C}_{2} \nsubseteq \overline{\mathcal{S}}_{0}$.

Lemma 22. $\mathcal{C}_{1} \cup \mathcal{D}_{1} \subsetneq \mathcal{C}_{2} \cap \mathcal{D}_{2}$, i.e., there is an iposet with two non-trivial different decompositions.

Proof. Directly from Lemma 14

Next we show that the $\mathcal{C}_{n}$ hierarchy is infinite, by exposing a sequence of witnesses for $\mathcal{C}_{2 n-1} \subsetneq \mathcal{C}_{2 n}$ for all $n \geq 1$.

Let $Q={ }^{0} \mathrm{id}_{1}^{0}, P_{1}=Q \triangleright Q$, and for $n \geq 1, P_{n+1}=Q \triangleright\left(P_{n} \otimes P_{n}\right)$. Note that all these are series-parallel posets. Graphically:

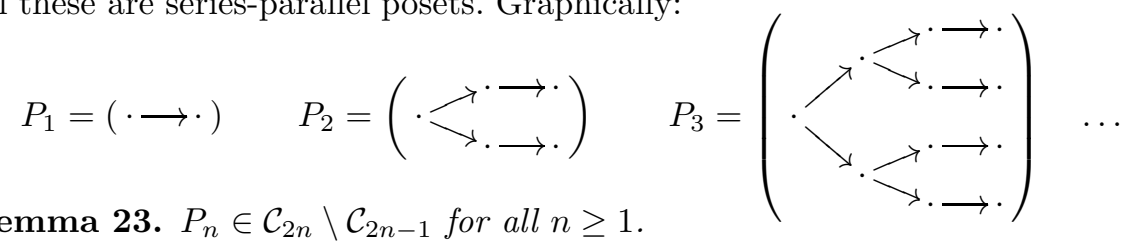

Proof. By induction. For $n=1, P_{1} \notin \mathcal{C}_{1}$, but $Q \in \mathcal{C}_{0} \subseteq \mathcal{C}_{1}$ and hence $P_{1}=$ $Q \triangleright Q \in \mathcal{C}_{2}=\mathcal{C}_{1}^{\triangleright}$.

Now for $n \geq 1$, suppose $C_{2 n-1} \not \ngtr P_{n} \in \mathcal{C}_{2 n}$. We use Lemma 15 to show that $P_{n} \otimes P_{n} \in \mathcal{C}_{2 n+1} \backslash \mathcal{C}_{2 n}$ : Obviously $P_{n} \otimes P_{n} \in \mathcal{C}_{2 n+1}=\mathcal{C}_{2 n}^{\otimes}$. If $P_{n} \otimes P_{n} \in \mathcal{C}_{2 n}=$ $\mathcal{C}_{2 n-1}^{\triangleright}$, then $P_{n} \otimes P_{n}=Q_{1} \triangleright \cdots \triangleright Q_{k}$ for some $Q_{1}, \ldots, Q_{k} \in \mathcal{C}_{2 n-1}$. Yet $P_{n} \notin \mathcal{C}_{1}$, which contradicts Lemma 15 .

Now to $P_{n+1}=Q \triangleright\left(P_{n} \otimes P_{n}\right)$. Trivially, $P_{n+1} \in \mathcal{C}_{2 n+2}=\mathcal{C}_{2 n+1}^{\triangleright}$. Suppose $P_{n+1} \in \mathcal{C}_{2 n+1}=\mathcal{C}_{2 n}^{\otimes} . P_{n+1}$ is connected, hence not a parallel product, so that $P_{n+1}$ must already be in $\mathcal{C}_{2 n}=\mathcal{C}_{2 n-1}^{\triangleright}$ and therefore $P_{n+1}=R_{1} \triangleright R_{2}$. Then, by Levi's lemma, there is an iposet $S$ such that either $Q=R_{1} \triangleright S$ and $S \triangleright\left(P_{n} \otimes P_{n}\right)=$ $R_{2}$ or $R_{1}=Q \triangleright S$ and $S \triangleright R_{2}=P_{2} \otimes P_{n}$. In the second case, $S \triangleright R_{2}=P_{2} \otimes P_{n}$, which again contradicts Lemma 15] in the first case, both $R_{1}$ and $S$ must be single points (with suitable interfaces), so that either $R_{1}={ }^{0} \mathrm{id}_{1}^{1}$ and $R_{2}=P_{n+1}$ (with an extra starting interface) or $R_{1}=Q$ and $R_{2}=P_{n} \otimes P_{n}$. This shows that $P_{n+1}=Q \triangleright\left(P_{n} \otimes P_{n}\right)$ is the only non-trivial $\triangleright$-decomposition of $P_{n+1}$. Thus $P_{n} \in \mathcal{C}_{2 n-1}$, a contradiction, and therefore $P_{n+1} \notin \mathcal{C}_{2 n+1}$.

Corollary 24. $\mathcal{C}_{2 n-1} \subsetneq \mathcal{C}_{2 n}$ for all $n \geq 1$, hence the $\mathcal{C}_{n}$ hierarchy does not collapse, and neither does the $\mathcal{D}_{n}$ hierarchy.

Proof. The last statement follows from $\mathcal{D}_{2 n-2} \subseteq \mathcal{C}_{2 n-1} \subsetneq \mathcal{C}_{2 n} \subseteq \mathcal{D}_{2 n+1}$.

Corollary 25. For all $n \in \mathbb{N}, \overline{\mathcal{S}}_{0} \nsubseteq \mathcal{C}_{n}$ and $\overline{\mathcal{S}}_{0} \nsubseteq \mathcal{D}_{n}$.

Proof. As we have already noted above, $P_{n} \in \overline{\mathcal{S}}_{0}$ for all $n$, which together with Lemma 23 implies the first statement. The second follows from $\mathcal{C}_{n} \subseteq \mathcal{D}_{n+1}$.

We have seen that the $\mathcal{C}_{n}$ and $\mathcal{D}_{n}$ hierarchies are properly infinite and that they contain the set of sp-posets only in the limit $\overline{\mathcal{S}}=\bigcup_{n \geq 0} \mathcal{C}_{n}=\bigcup_{n \geq 0} \mathcal{D}_{n}$.

Finally, we turn to the question of characterising this limit $\overline{\mathcal{S}}$ geometrically. Recalling that a poset is series-parallel iff if it does not contain an induced subposet isomorphic to $\mathrm{N}$, we would like a similar characterisation using forbidden 
subposets for the gp-(i)posets. We expose five such forbidden subposets, but leave the question of whether there are others to future work.

Define the following five posets on six points:

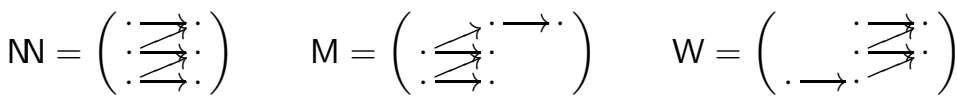

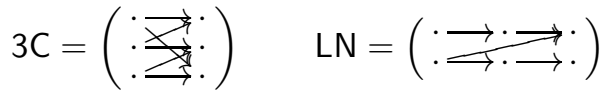

Proposition 26. If $P \in \overline{\mathcal{S}}$, then $P$ does not contain $N, M, W, 3 C$, or $L N$ as induced subposets.

Proof. We only show the proof for $\mathrm{N}$; the others are very similar and are left to the reader. We can assume that $P$ is connected. We use structural induction, noting that all $P \in \mathcal{S}$ are $\mathrm{N}$-free, so it remains to show that $P \triangleright Q$ is $\mathrm{N}$-free

whenever $P$ and $Q$ are.
By contraposition, suppose $P \triangleright Q$ contains the induced sub-N $\left(\begin{array}{l}a \longrightarrow b \\ c \rightrightarrows d \\ e \longrightarrow f\end{array}\right)$.
Then we show that either $P$ or $Q$ also have an induced sub-N.

Assume first that $a \in Q$. Then $a \leq_{Q} b$, hence also $b \in Q$, but $b \notin Q_{\min }$, that is, $b \notin s_{Q}$. Now $e \mathbb{X}_{P \triangleright Q} b$, which forces $e \in t_{P}$ and therefore in $e \in Q$. This in turn implies that $d, f \in Q$ and in particular $e \leq_{Q} f$. Thus $f \notin Q_{\min }$ and therefore $f \notin s_{Q}$, which forces $c \in t_{P}$ and therefore $c \in Q$. This shows that $\mathrm{N}$ lies entirely in $Q$.

Finally assume that $a \notin Q$. Then $a \in P \backslash t_{P}$, and as $a \not \mathbb{P}_{P \triangleright Q} d$ and $a \not \mathbb{P}_{P \triangleright Q} f$, we must have $d, f \in s_{Q}$ and therefore $d, f \in P$. This forces $c, e \in P$ and in particular $e \leq_{P} f$. Thus $e \notin P_{\min }$, whence $e \notin t_{P}$. This in turn forces $b \in s_{Q}$ and therefore $b \in P$. This shows that $\mathrm{N}$ lies entirely in $P$.

\section{Experiments}

We have encoded most of the constructions in this paper with Python to experiment with gluing-parallel (i)posets. Notably, Proposition 26 is, in part, a result of these experiments 6 Our prototype is rather inefficient, which explains why some numbers are "n.a.", i.e., not available, in Table 1

Using procedures to generate non-isomorphic posets of different types, we have used our software to verify that

1. all posets on five points are in $\overline{\mathcal{S}}$, i.e., gp-posets;

2. N, M, W, 3C, and LN are the only six-point posets that are not in $\overline{\mathcal{S}}$.

We provide tables of gluing-parallel decompositions of posets in appendix to prove these claims.

We have also used our software to count non-isomorphic posets and iposets of different types, see Table 1. We note that P and SP are sequences no. A000112 
Table 1. Different types of posets with $n$ points: all posets; sp-posets; gp-posets; (weakly) connected gp-posets; iposets with starting interfaces only; iposets; gp-iposets.

\begin{tabular}{r|rrrrrrr}
$n$ & $\mathrm{P}(n)$ & $\mathrm{SP}(n)$ & $\mathrm{GP}(n)$ & $\mathrm{GPC}(n)$ & $\mathrm{SIP}(n)$ & $\mathrm{IP}(n)$ & $\mathrm{GPI}(n)$ \\
\hline 0 & 1 & 1 & 1 & 1 & 1 & 1 & 1 \\
1 & 1 & 1 & 1 & 1 & 2 & 4 & 4 \\
2 & 2 & 2 & 2 & 1 & 5 & 17 & 16 \\
3 & 5 & 5 & 5 & 3 & 16 & 86 & 74 \\
4 & 16 & 15 & 16 & 10 & 66 & 532 & 419 \\
5 & 63 & 48 & 63 & 44 & 350 & n.a. & 2980 \\
6 & 318 & 167 & 313 & 233 & n.a. & n.a. & 26566
\end{tabular}

and A003430, respectively, in the On-Line Encyclopedia of Integer Sequences (OEIS) 7 Sequences GPC, SIP, IP, and GPI are unknown to the OEIS.

The single iposet on two points which is not gluing-parallel is the symmetry $[2]: 2 \rightarrow 2$ with $s(1)=1, s(2)=2, t(1)=2$, and $t(2)=1$. The prefix of GP we were able to compute equals the corresponding prefix of sequence no. A079566 in the OEIS, 7 which counts the number of connected (undirected) graphs which have no induced 4-cycle $C_{4}$. We leave it to the reader to ponder upon the relation between gp-posets and $C_{4}$-free connected graphs.

\section{References}

1. S. L. Bloom and Z. Ésik. Free shuffle algebras in language varieties. Theor. Comput. Sci., 163(1\&2):55-98, 1996.

2. J. H. Conway. Regular Algebra and Finite Machines. Chapman and Hall, 1971.

3. B. Courcelle and J. Engelfriet. Graph Structure and Monadic Second-Order Logic - A Language-Theoretic Approach. Cambridge University Press, 2012.

4. M. P. Fiore and M. D. Campos. The algebra of directed acyclic graphs. In Computation, Logic, Games, and Quantum Foundations, vol. 7860 of LNCS. Springer, 2013.

5. P. C. Fishburn. Intransitive indifference with unequal indifference intervals. $J$. Math. Psych., 7(1):144-149, 1970.

6. P. C. Fishburn. Interval Orders and Interval Graphs: A Study of Partially Ordered Sets. Wiley, 1985.

7. H. Furusawa and G. Struth. Concurrent dynamic algebra. ACM Trans. Comput. Log., 16(4):30:1-30:38, 2015.

8. J. L. Gischer. The equational theory of pomsets. Theor. Comput. Sci., 61:199-224, 1988.

9. M. C. Golumbic and A. N. Trenk. Tolerance Graphs. Cambridge University Press, 2004.

10. J. Grabowski. On partial languages. Fund. Inf., 4(2):427, 1981.

11. M. Herlihy and J. M. Wing. Linearizability: A correctness condition for concurrent objects. ACM Trans. Program. Lang. Syst., 12(3):463-492, 1990.

\footnotetext{
${ }^{6}$ Our software is available at http://www.lix.polytechnique.fr/ uli/posets/

7 See http://oeis.org/A000112, oeis.org/A003430, and oeis.org/A079566
} 
12. T. Hoare, B. Möller, G. Struth, and I. Wehrman. Concurrent Kleene algebra and its foundations. J. Log. Algebr. Program., 80(6):266-296, 2011.

13. T. Hoare, S. van Staden, B. Möller, G. Struth, J. Villard, H. Zhu, and P. W. O'Hearn. Developments in concurrent Kleene algebra. In RAMiCS 2014, vol. 8428 of $L N C S$. Springer, 2014.

14. R. Janicki. Modeling operational semantics with interval orders represented by sequences of antichains. In PETRI NETS 2018, vol. 10877 of LNCS. Springer, 2018.

15. R. Janicki and M. Koutny. Structure of concurrency. Theor. Comput. Sci., 112(1):5-52, 1993.

16. R. Janicki and X. Yin. Modeling concurrency with interval traces. Inf. Comput., 253:78-108, 2017.

17. P. Jipsen and M. A. Moshier. Concurrent Kleene algebra with tests and branching automata. J. Log. Algebr. Meth. Program., 85(4):637-652, 2016.

18. T. Kappé, P. Brunet, J. Rot, A. Silva, J. Wagemaker, and F. Zanasi. Kleene algebra with observations. In CONCUR 2019, vol. 140 of LIPIcs. Schloss Dagstuhl - Leibniz-Zentrum für Informatik, 2019.

19. T. Kappé, P. Brunet, A. Silva, and F. Zanasi. Concurrent Kleene algebra: Free model and completeness. In ESOP 2018, vol. 10801 of LNCS. Springer, 2018.

20. L. Lamport. The mutual exclusion problem: Part I - a theory of interprocess communication. J. ACM, 33(2):313-326, 1986.

21. L. Lamport. On interprocess communication. Part I: basic formalism. Distributed Computing, 1(2):77-85, 1986.

22. M. R. Laurence and G. Struth. Completeness theorems for pomset languages and concurrent Kleene algebras. CoRR, abs/1705.05896, 2017.

23. F. W. Levi. On semigroups. Bull. Calcutta Math. Soc., 36:141-146, 1944.

24. K. Lodaya and P. Weil. Series-parallel languages and the bounded-width property. Theor. Comput. Sci., 237(1-2):347-380, 2000.

25. S. Mimram. Presenting finite posets. In TERMGRAPH 2014, vol. 183 of EPTCS, 2014.

26. B. Möller and T. Hoare. Exploring an interface model for CKA. In MPC 2015, vol. 9129 of $L N C S$. Springer, 2015.

27. B. Möller, T. Hoare, M. E. Müller, and G. Struth. A discrete geometric model of concurrent program execution. In UTP 2016, vol. 10134 of LNCS. Springer, 2016.

28. D. Peleg. Concurrent dynamic logic. J. ACM, 34(2):450-479, 1987.

29. J. Valdes, R. E. Tarjan, and E. L. Lawler. The recognition of series parallel digraphs. SIAM J. Comput., 11(2):298-313, 1982.

30. R. J. van Glabbeek. The refinement theorem for ST-bisimulation semantics. In IFIP TC2 Working Conf. Programming Concepts and Methods. North-Holland, 1990.

31. R. J. van Glabbeek and F. W. Vaandrager. Petri net models for algebraic theories of concurrency. In PARLE (2), vol. 259 of LNCS. Springer, 1987.

32. W. Vogler. Failures semantics based on interval semiwords is a congruence for refinement. Distributed Computing, 4:139-162, 1991.

33. W. Vogler. Modular Construction and Partial Order Semantics of Petri Nets, vol. 625 of Lecture Notes in Computer Science. Springer, 1992.

34. N. Wiener. A contribution to the theory of relative position. Proc. Camb. Philos. Soc., 17:441-449, 1914.

35. J. Winkowski. An algebraic characterization of the behaviour of non-sequential systems. Inf. Process. Lett., 6(4):105-109, 1977. 


\section{Appendix}

The following tables show gluing-parallel decompositions of all (weakly) connected posets on four points, all connected posets on five points, and all connected posets on six points except for the five posets $\mathrm{N}, \mathrm{M}, \mathrm{W}, 3 \mathrm{C}$, and $\mathrm{LN}$ which are not gluing-parallel.

Given that disconnected posets can be decomposed into posets with fewer points using $\otimes$ and that all posets on fewer than four points are series-parallel, hence gluing-parallel, these tables show the claims in Section 7 All posets on five points are gluing-parallel, as are all but the five exceptional posets $\mathrm{N}, \mathrm{M}$, W, 3C, and LN on six points.

Table 2: Gluing-Parallel decompositions of connected posets on four points

\begin{tabular}{|c|c|c|c|}
\hline no. & Poset & \multicolumn{2}{|c|}{ Decomposition } \\
\hline 1 & 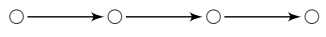 & $\mathrm{O} \longrightarrow \mathrm{O}$ & $\circ \longrightarrow 0$ \\
\hline 2 & $\rightarrow 0$ & $\circ \longrightarrow 0$ & $\begin{array}{l}0 \\
0\end{array}$ \\
\hline 3 & - & $\begin{array}{l}0 \\
0\end{array}$ & 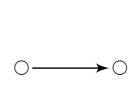 \\
\hline 4 & & $\begin{array}{l}\circ \\
\circ\end{array}$ & $\begin{array}{l}0 \\
0\end{array}$ \\
\hline 5 & & o & \\
\hline 6 & $\circ \overline{-}$ & 0 & $\mathrm{O} \longrightarrow \mathrm{O}$ \\
\hline 7 & & o & $\begin{array}{l}0 \\
0 \\
0\end{array}$ \\
\hline 8 & $\longrightarrow$ & $\begin{array}{l}0 \\
\mathbf{1}\end{array}$ & $\longrightarrow^{\circ}$ \\
\hline 9 & $\longrightarrow_{0}^{\circ \longrightarrow}$ & $\begin{array}{l} \\
\mathbf{1}^{1}\end{array}$ & $>_{1}^{0}$ \\
\hline 10 & $0-$ & $\begin{array}{l}0 \\
0 \\
0\end{array}$ & 0 \\
\hline
\end{tabular}


Table 3: Gluing-parallel decompositions of connected posets on five points

\begin{tabular}{|c|c|c|c|}
\hline no. & Poset & \multicolumn{2}{|c|}{ Decomposition } \\
\hline 1 & $\mathrm{O} \longrightarrow \mathrm{O} \longrightarrow \mathrm{O} \longrightarrow \mathrm{O} \longrightarrow \mathrm{O}$ & $\circ \longrightarrow 0$ & $\mathrm{\longrightarrow} \longrightarrow \mathrm{O}$ \\
\hline 2 & $\bar{x}_{0}^{0}$ & $\circ \longrightarrow \circ$ & $\searrow_{0}^{\circ}$ \\
\hline 3 & & ○ & \\
\hline 4 & $\mathrm{\longrightarrow}$ & $\circ \longrightarrow \circ$ & ○ \\
\hline 5 & o- & $\mathrm{O} \longrightarrow \mathrm{O}$ & $\begin{array}{l}0 \\
0 \\
0\end{array}$ \\
\hline 6 & 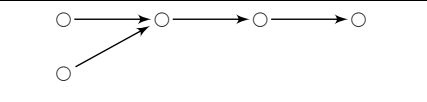 & $\begin{array}{l}0 \\
0\end{array}$ & $\circ \longrightarrow 0 \longrightarrow 0$ \\
\hline 7 & & $\begin{array}{l}0 \\
0\end{array}$ & $\searrow_{0}^{\circ}$ \\
\hline 8 & & $\begin{array}{l}\circ \\
\circ\end{array}$ & $\longrightarrow^{\circ}$ \\
\hline 9 & 0 & $\begin{array}{l}0 \\
0\end{array}$ & $\mathrm{O} \longrightarrow$ \\
\hline 10 & & $\begin{array}{l}\circ \\
\circ\end{array}$ & $\begin{array}{l}0 \\
0 \\
0\end{array}$ \\
\hline 11 & & & $\mathrm{O} \longrightarrow \mathrm{O}$ \\
\hline 12 & & $\mathrm{X}_{0}^{0}$ & 0 \\
\hline 13 & $\longrightarrow 0$ & $\begin{array}{l}\mathrm{O} \longrightarrow \mathrm{O} \\
\mathrm{O}\end{array}$ & $0 \longrightarrow 0$ \\
\hline 14 & $\longrightarrow{ }^{\circ \longrightarrow} \longrightarrow 0$ & $\begin{array}{l}\mathrm{O} \longrightarrow \mathrm{O} \\
\mathrm{O}\end{array}$ & $\begin{array}{l}0 \\
0\end{array}$ \\
\hline 15 & $\mathrm{O} \mathrm{y}^{\mathrm{O}} \longrightarrow \mathrm{O}$ & $\begin{array}{l}0 \\
\circ \\
0\end{array}$ & $\mathrm{O} \longrightarrow \mathrm{O}$ \\
\hline
\end{tabular}


Table 3: Gluing-parallel decompositions of connected posets on five points

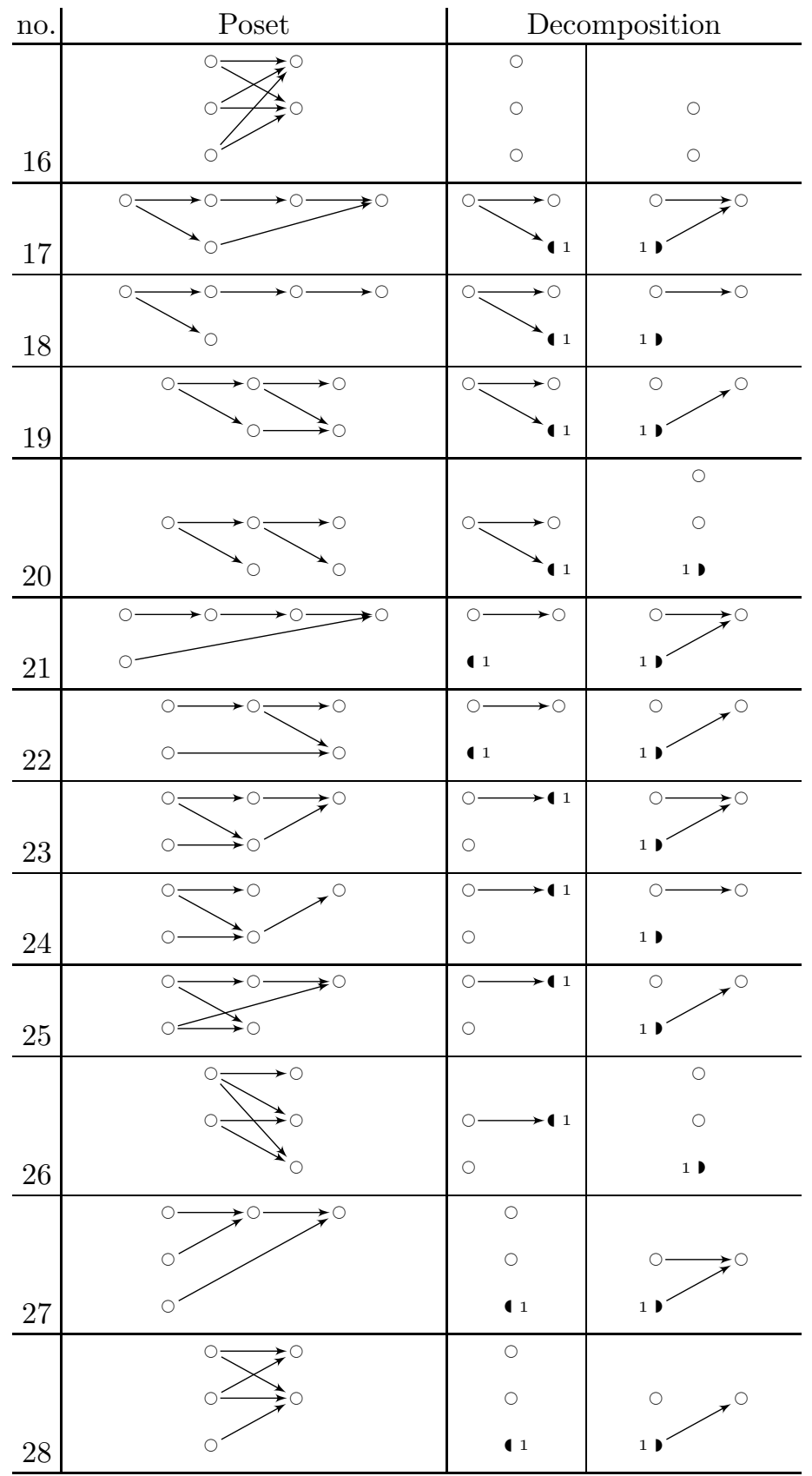


Table 3: Gluing-parallel decompositions of connected posets on five points

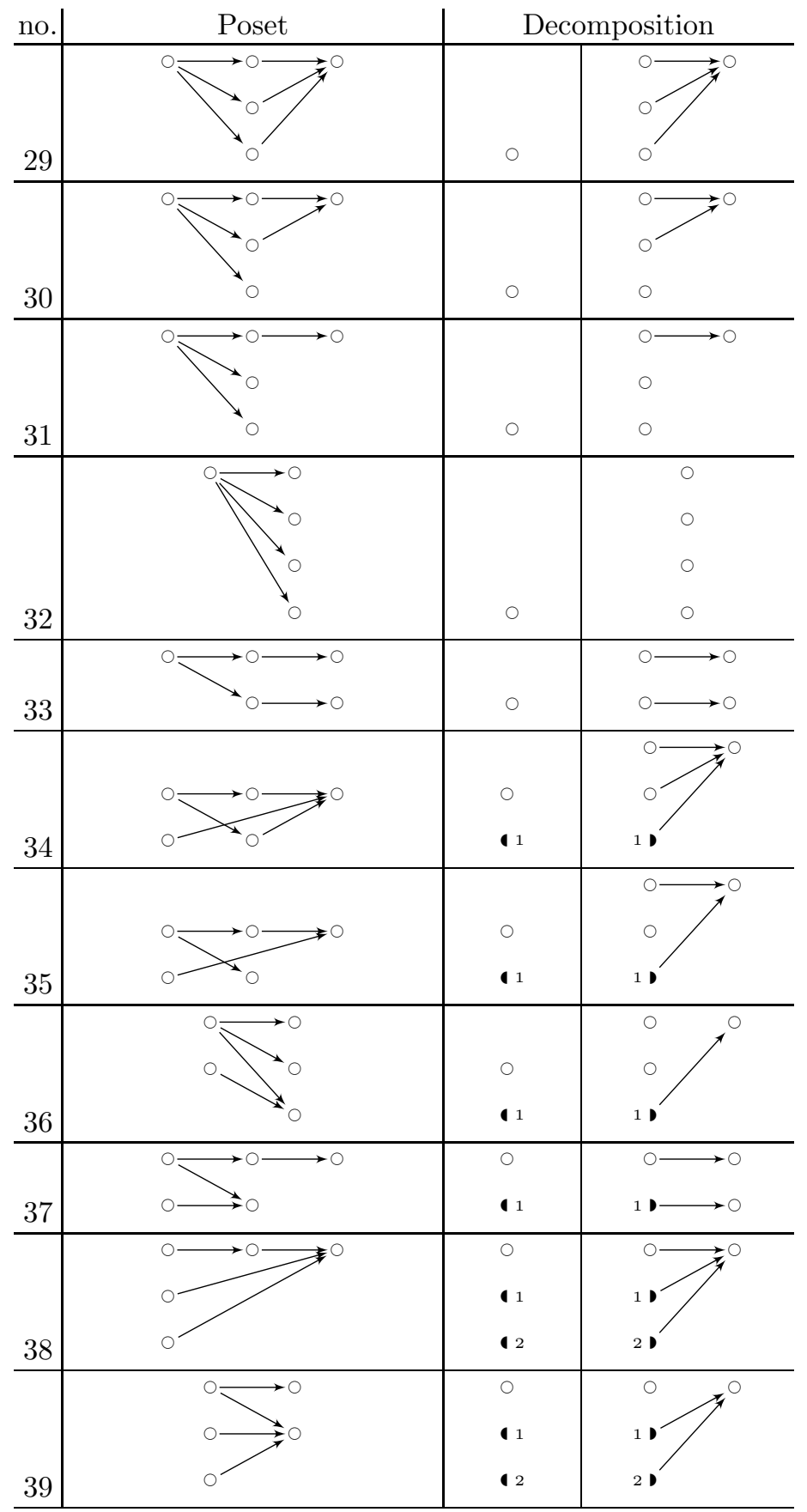


Table 3: Gluing-parallel decompositions of connected posets on five points

\begin{tabular}{|c|c|c|c|}
\hline no. & Poset & \multicolumn{2}{|c|}{ Decomposition } \\
\hline 40 & & $\begin{array}{l}0 \\
\mathbf{a}^{1} \\
\mathbf{a}^{2} \\
\end{array}$ & $\begin{array}{l}1 \bullet \longrightarrow 0 \\
2 \square \longrightarrow 0\end{array}$ \\
\hline 41 & & $\begin{array}{l}0 \\
0 \\
0 \\
0\end{array}$ & 0 \\
\hline 42 & $\circ$ & $\begin{array}{l}\circ \longrightarrow 0 \\
\circ \longrightarrow 0\end{array}$ & 0 \\
\hline 43 & $\stackrel{\longrightarrow}{\longrightarrow}$ & $\begin{array}{l}\circ \longrightarrow 0 \\
\circ \longrightarrow \mathbf{1} 1\end{array}$ & $\begin{array}{r}0 \\
1,\end{array}$ \\
\hline 44 & & $\begin{array}{l}\mathrm{O} \\
\mathrm{O} 1\end{array}$ & $\begin{array}{l} \\
1 \text { ग } \\
2 \text { । } \\
\end{array}$ \\
\hline
\end{tabular}

Table 4: Gluing-parallel decompositions of connected gp-posets on six points

\begin{tabular}{|c|c|c|c|}
\hline no. & Poset & \multicolumn{2}{|c|}{ Decomposition } \\
\hline 1 & $\mathrm{O} \longrightarrow \mathrm{O} \longrightarrow \mathrm{O} \longrightarrow \circ \longrightarrow \circ \longrightarrow \circ$ & $\mathrm{O} \longrightarrow \mathrm{O} \longrightarrow 0$ & $\mathrm{O} \longrightarrow 0 \longrightarrow 0$ \\
\hline 2 & 0 & 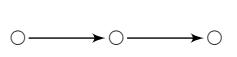 & 0 \\
\hline 3 & & $\circ \longrightarrow 0$ & \\
\hline 4 & $\longrightarrow$ & $\mathrm{O} \longrightarrow \mathrm{O} \longrightarrow 0$ & ○ \\
\hline 5 & $\circ \longrightarrow \circ \longrightarrow \circ \curvearrowright$ & $\mathrm{O} \longrightarrow \mathrm{O} \longrightarrow \mathrm{O}$ & $\begin{array}{l}0 \\
0 \\
\circ\end{array}$ \\
\hline 6 & & 0 & $\circ \longrightarrow 0 \longrightarrow 0$ \\
\hline 7 & & ○ & \\
\hline
\end{tabular}


Table 4: Gluing-parallel decompositions of connected gp-posets on six points

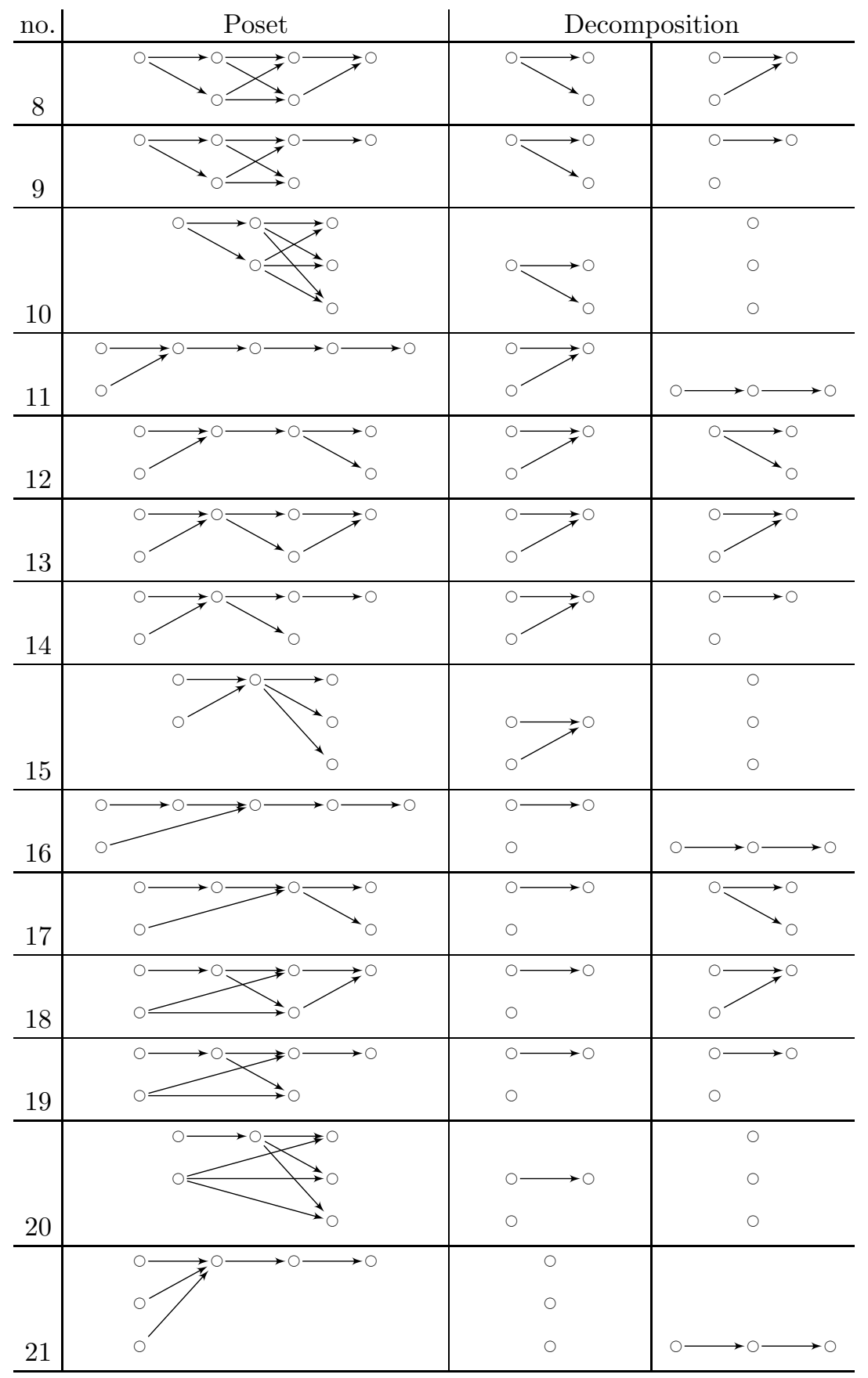


Table 4: Gluing-parallel decompositions of connected gp-posets on six points

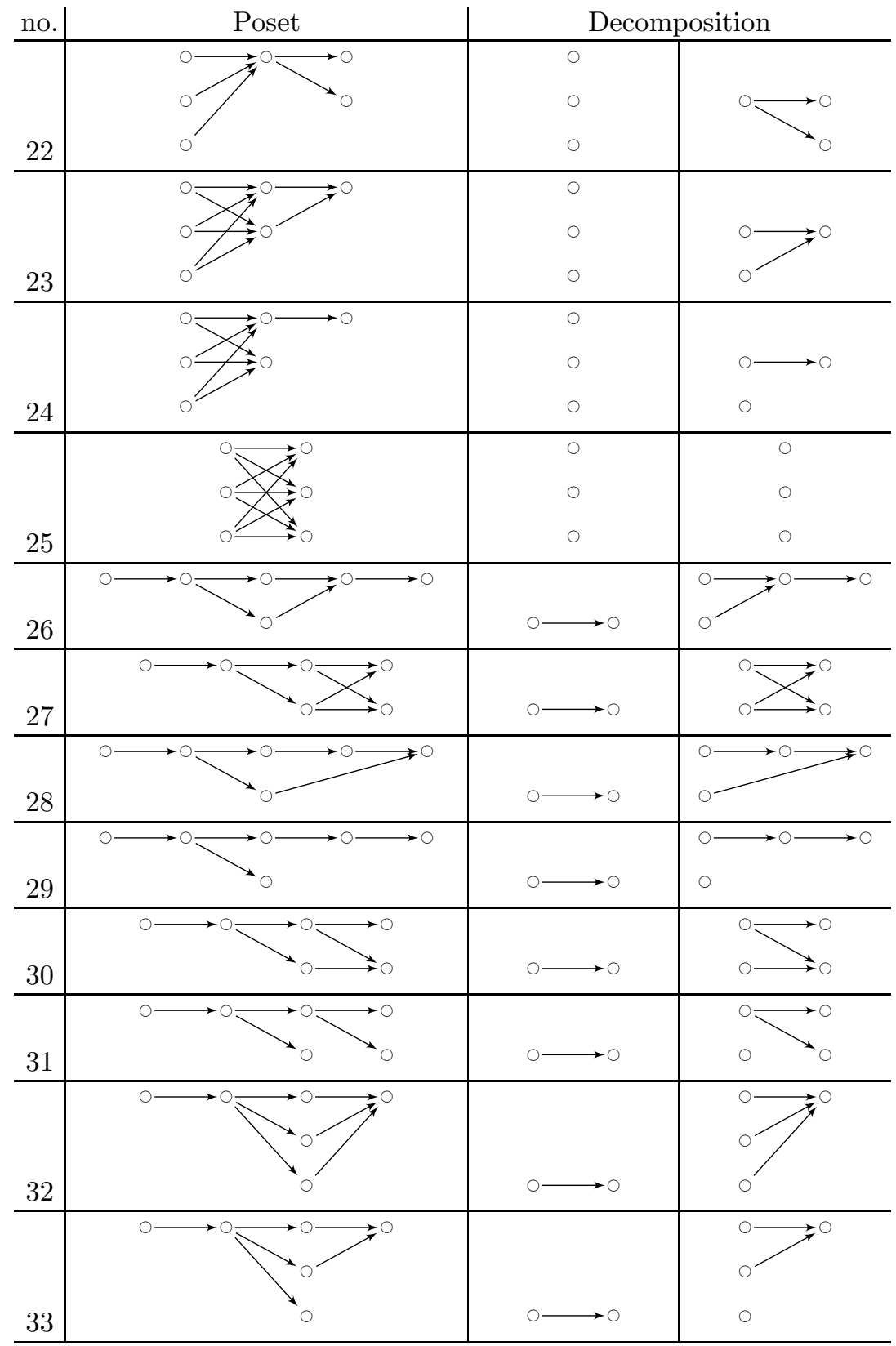


Table 4: Gluing-parallel decompositions of connected gp-posets on six points

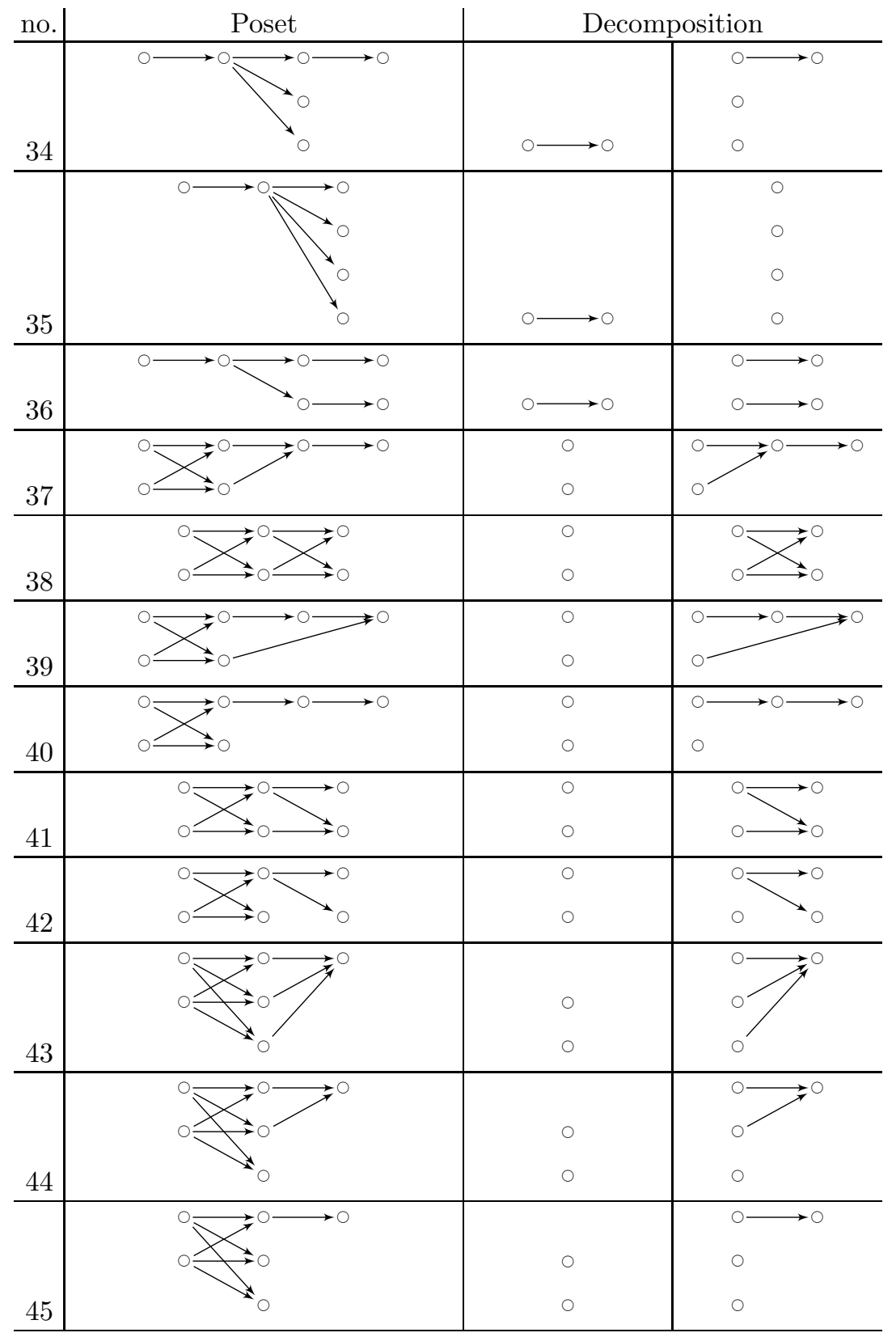


Table 4: Gluing-parallel decompositions of connected gp-posets on six points

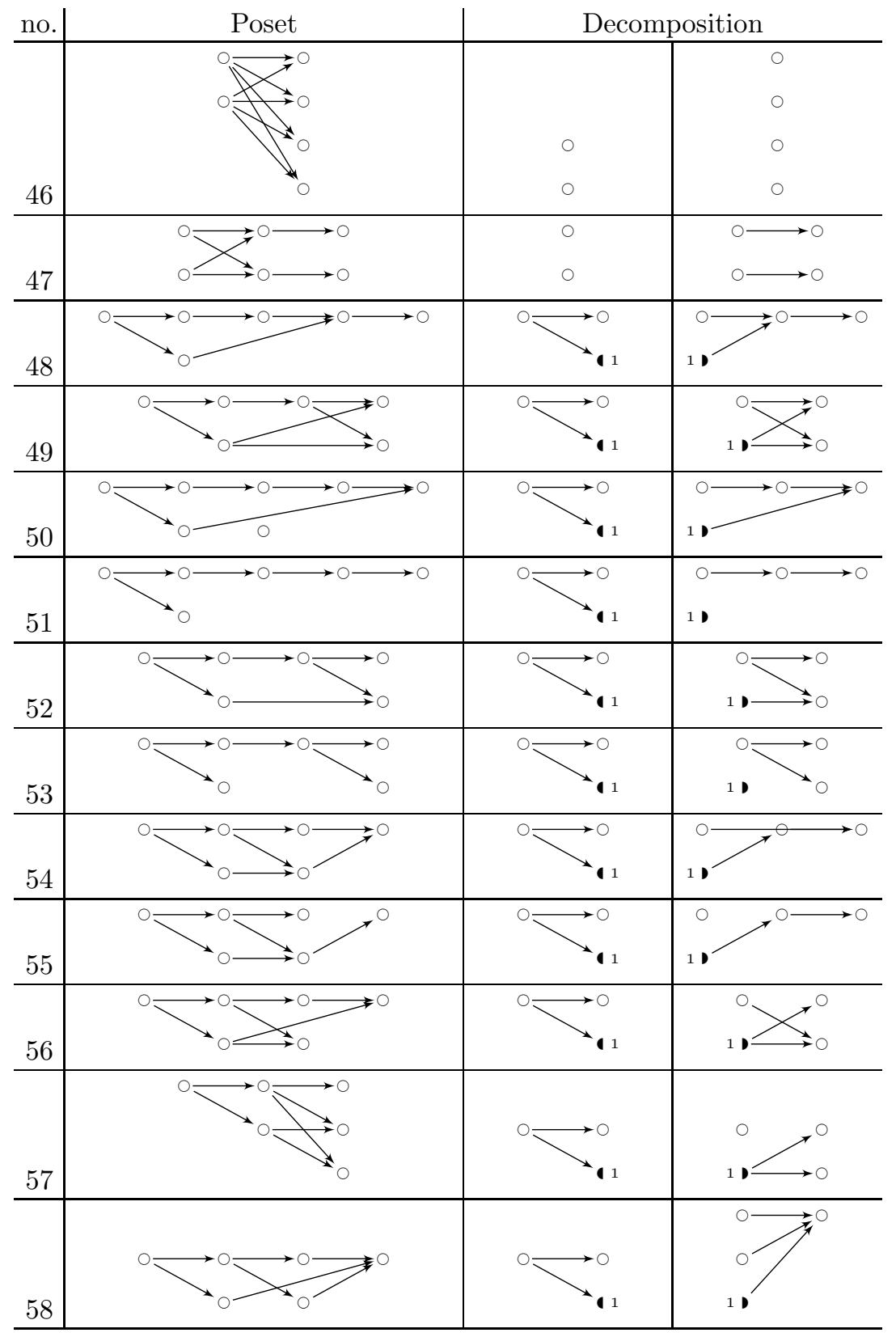


Table 4: Gluing-parallel decompositions of connected gp-posets on six points

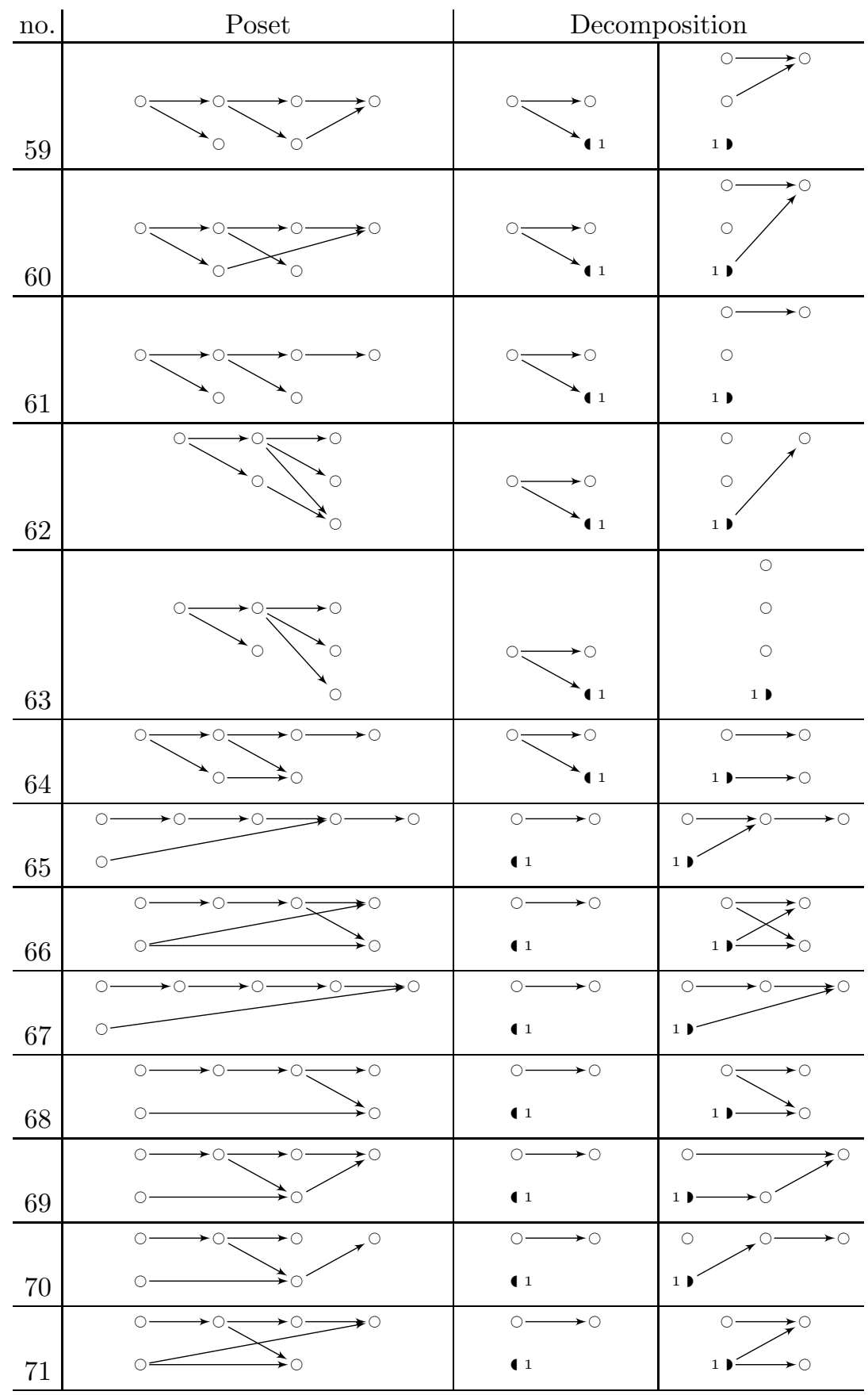


Table 4: Gluing-parallel decompositions of connected gp-posets on six points

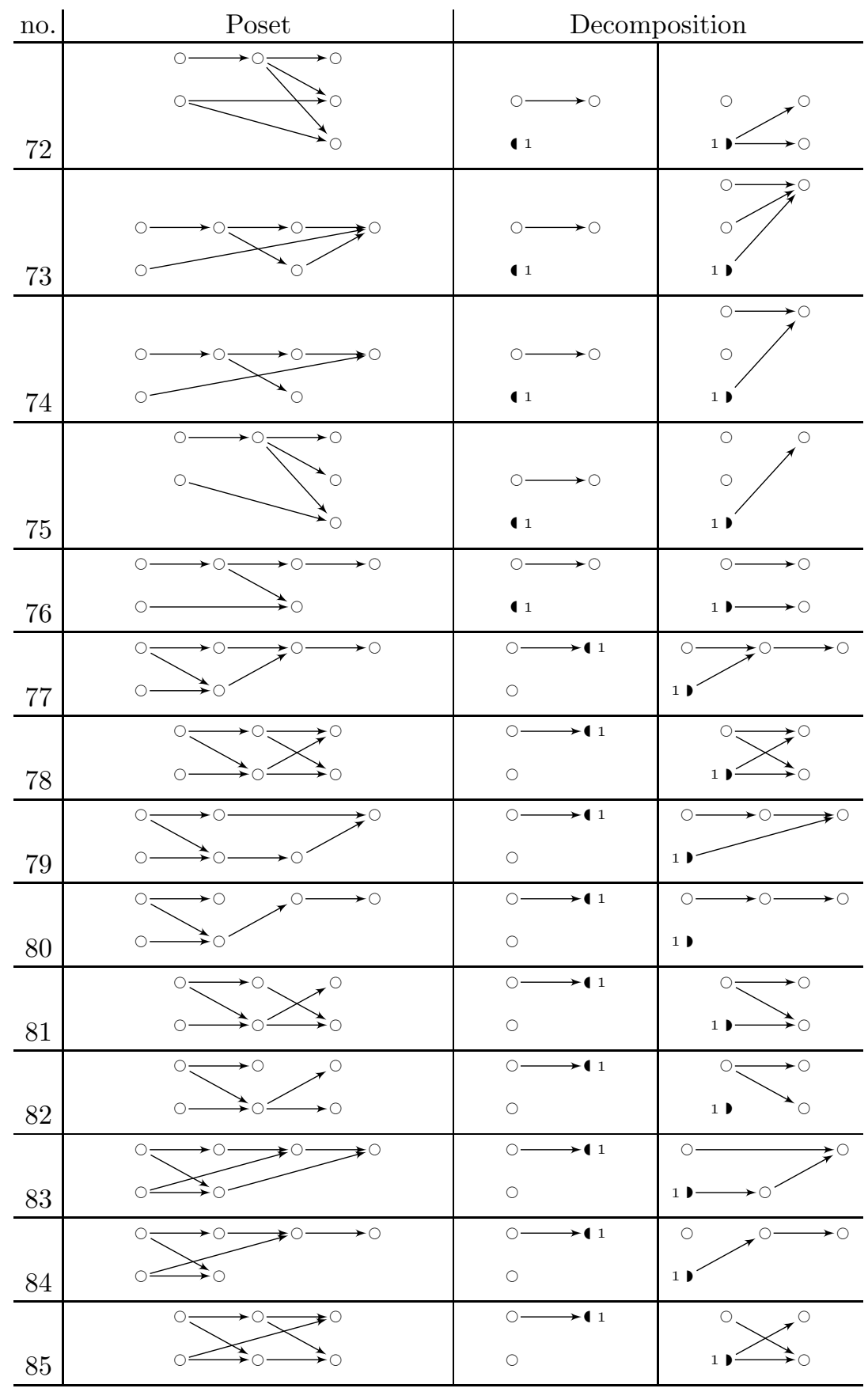


Table 4: Gluing-parallel decompositions of connected gp-posets on six points

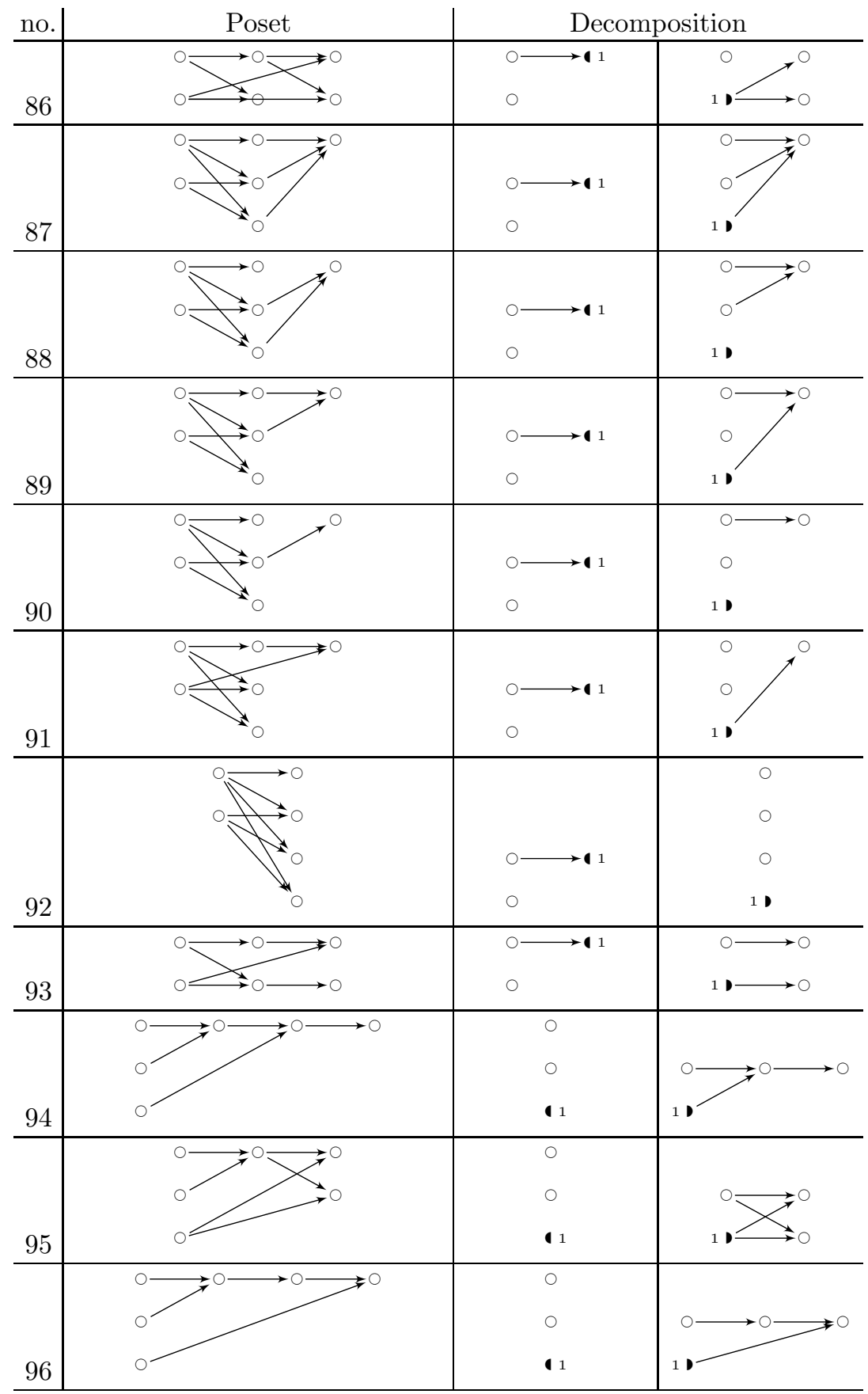


Table 4: Gluing-parallel decompositions of connected gp-posets on six points

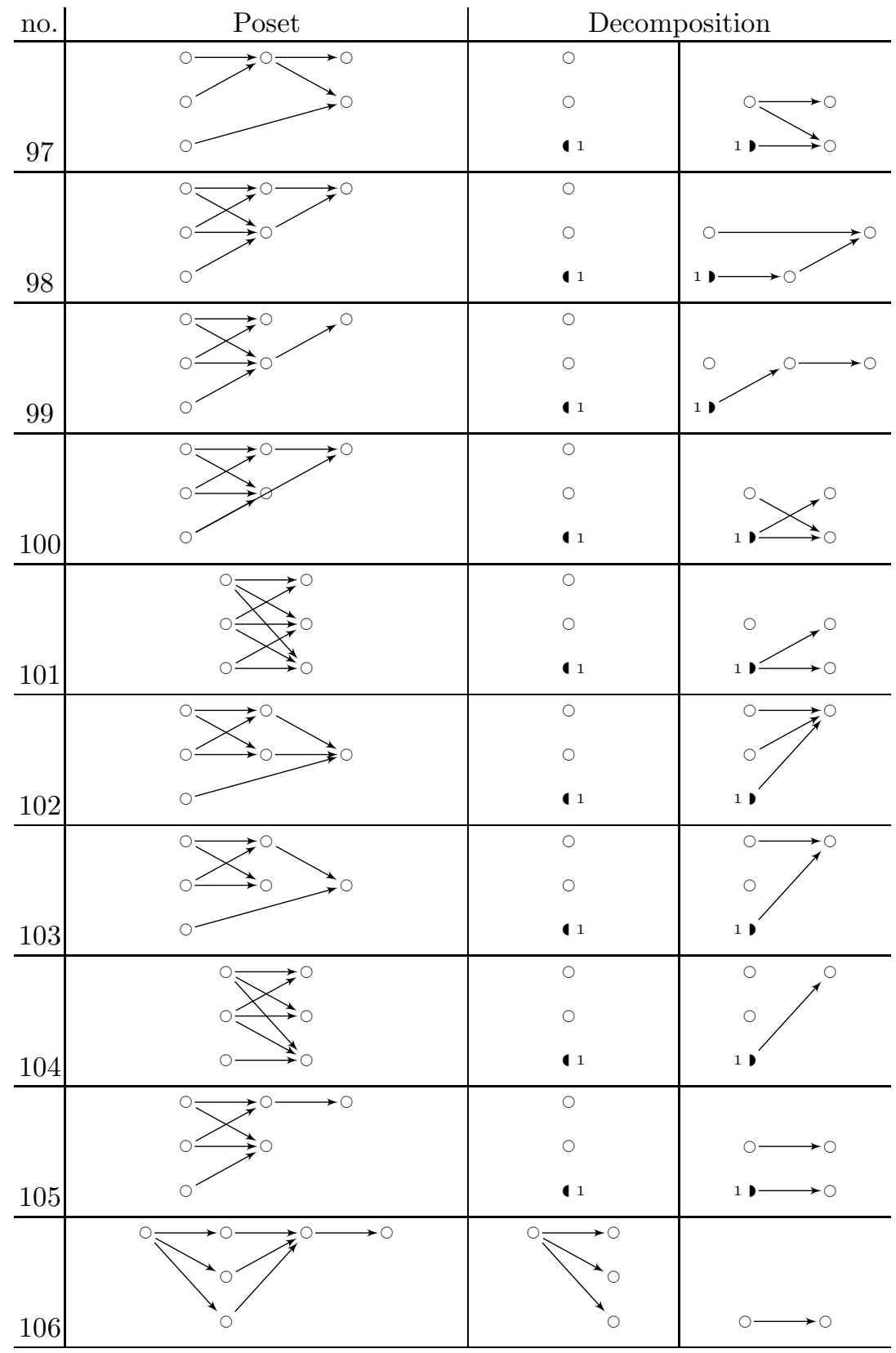


Table 4: Gluing-parallel decompositions of connected gp-posets on six points

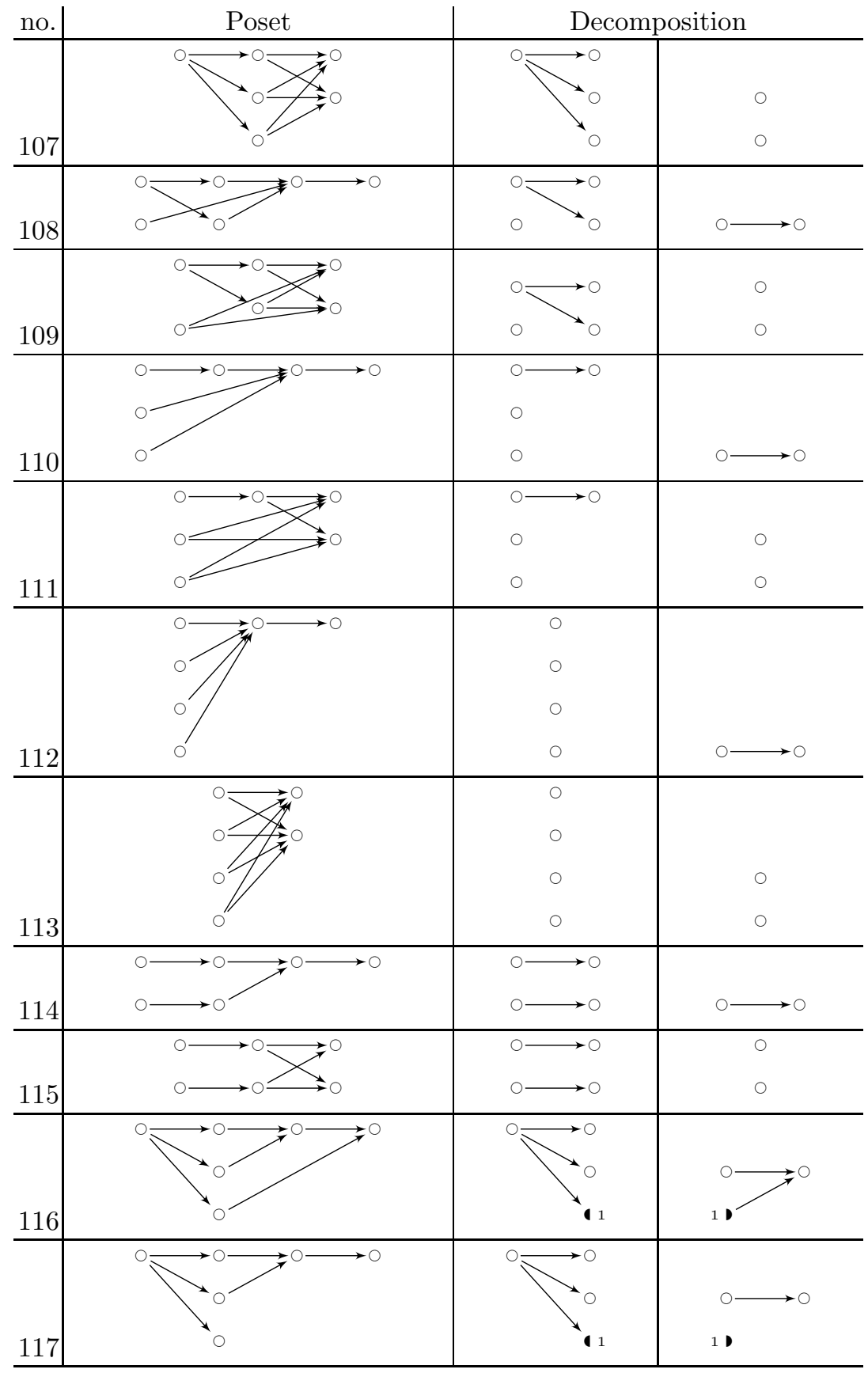


Table 4: Gluing-parallel decompositions of connected gp-posets on six points

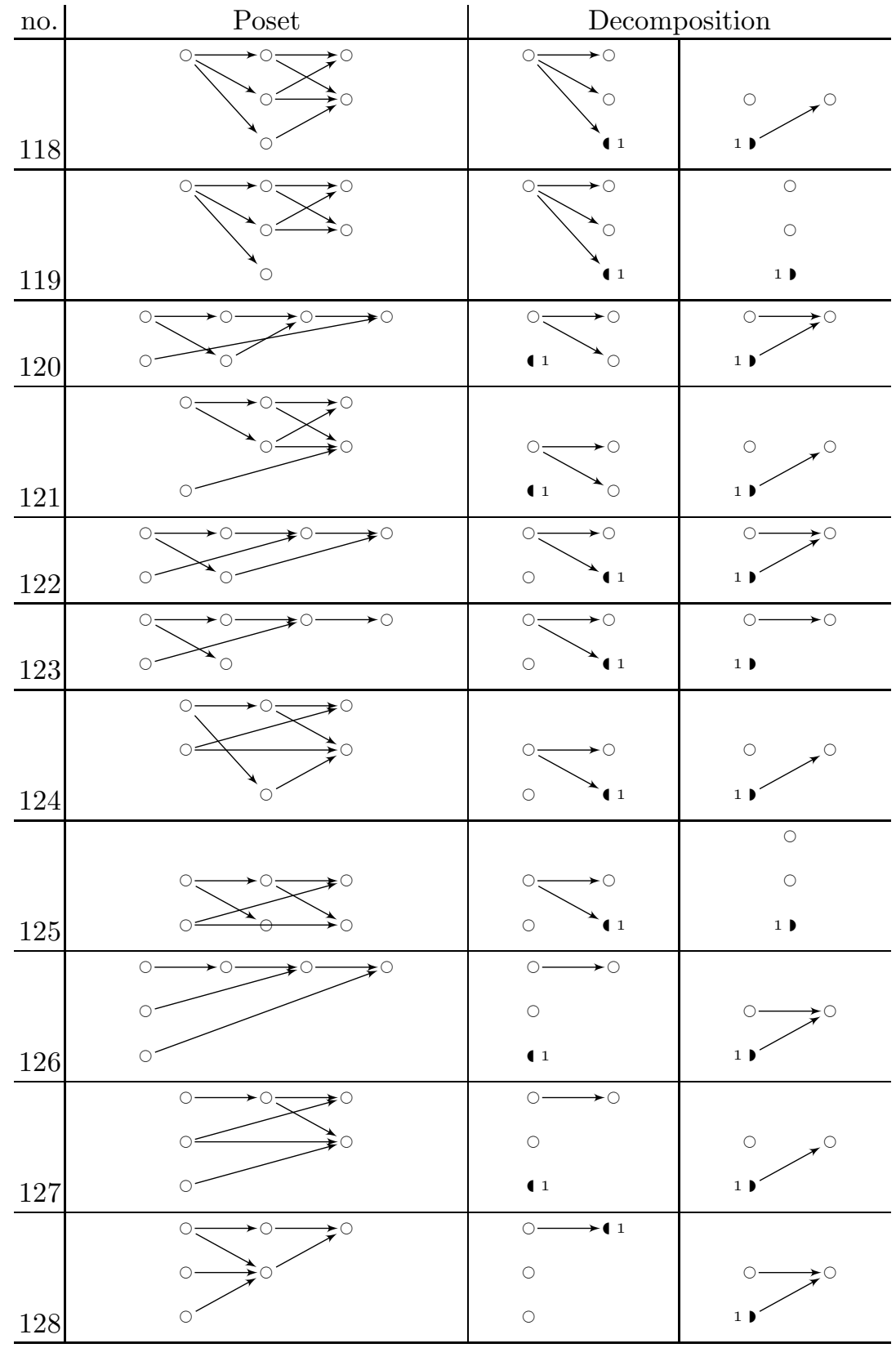


Table 4: Gluing-parallel decompositions of connected gp-posets on six points

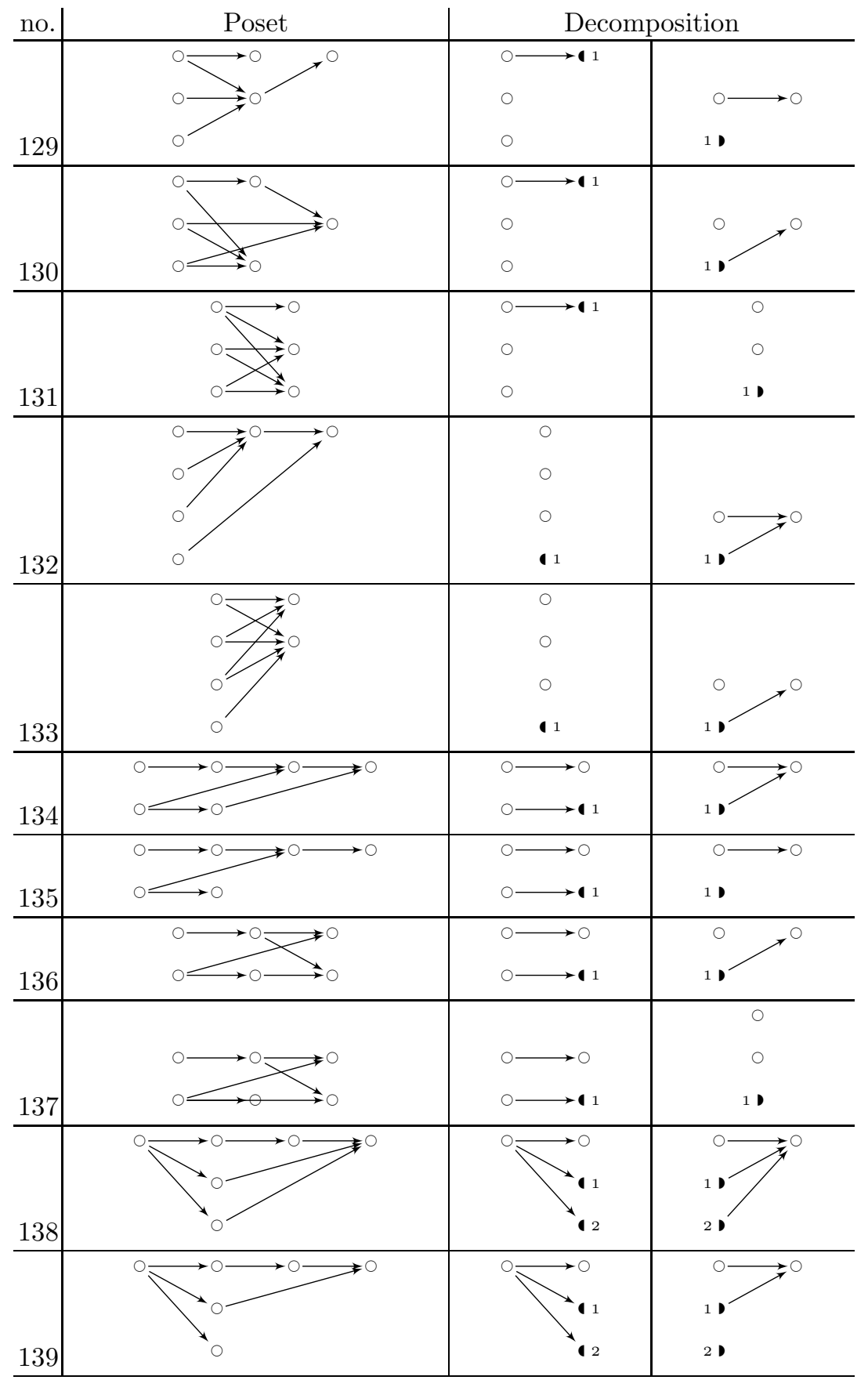


Table 4: Gluing-parallel decompositions of connected gp-posets on six points

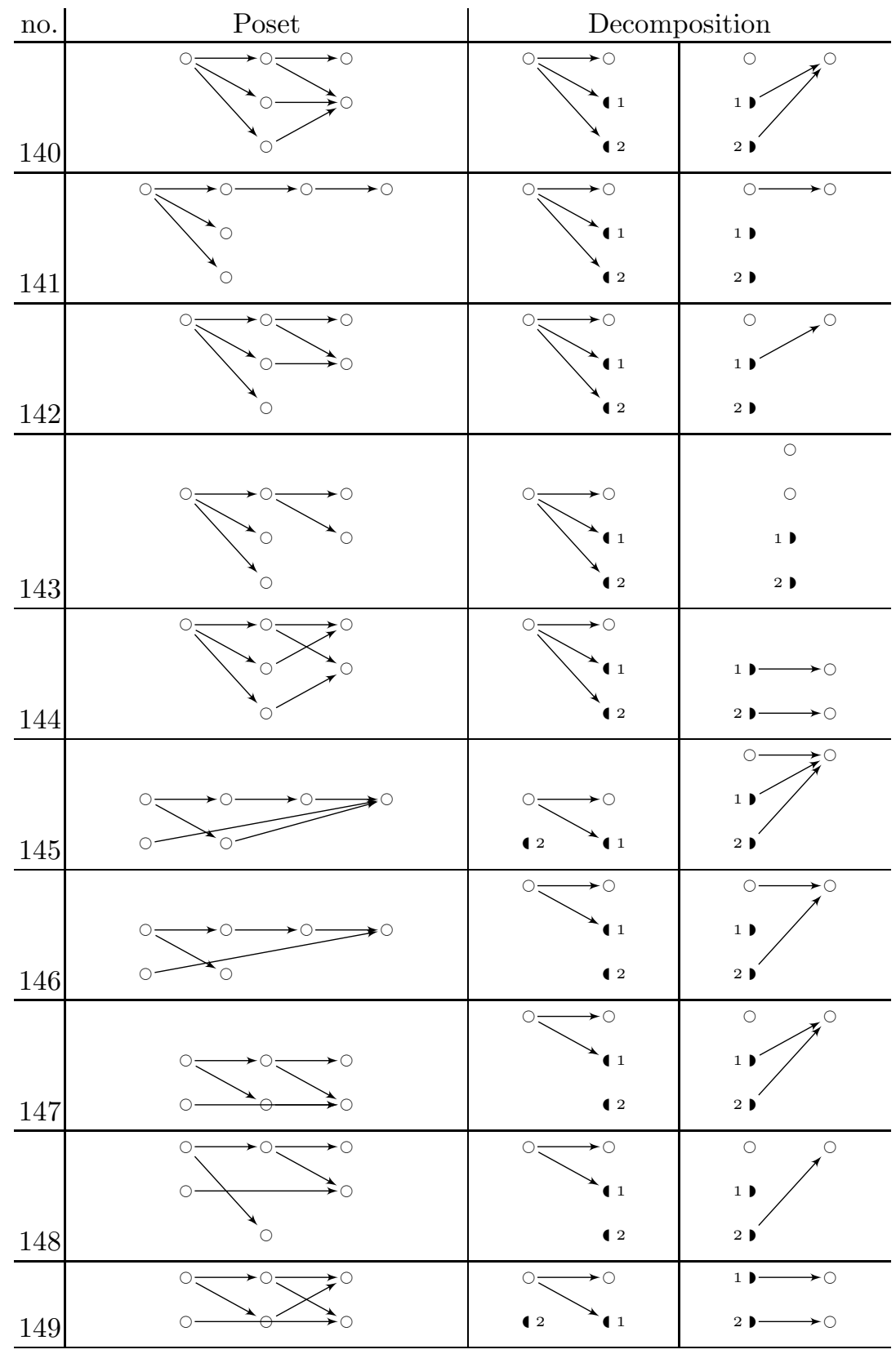


Table 4: Gluing-parallel decompositions of connected gp-posets on six points

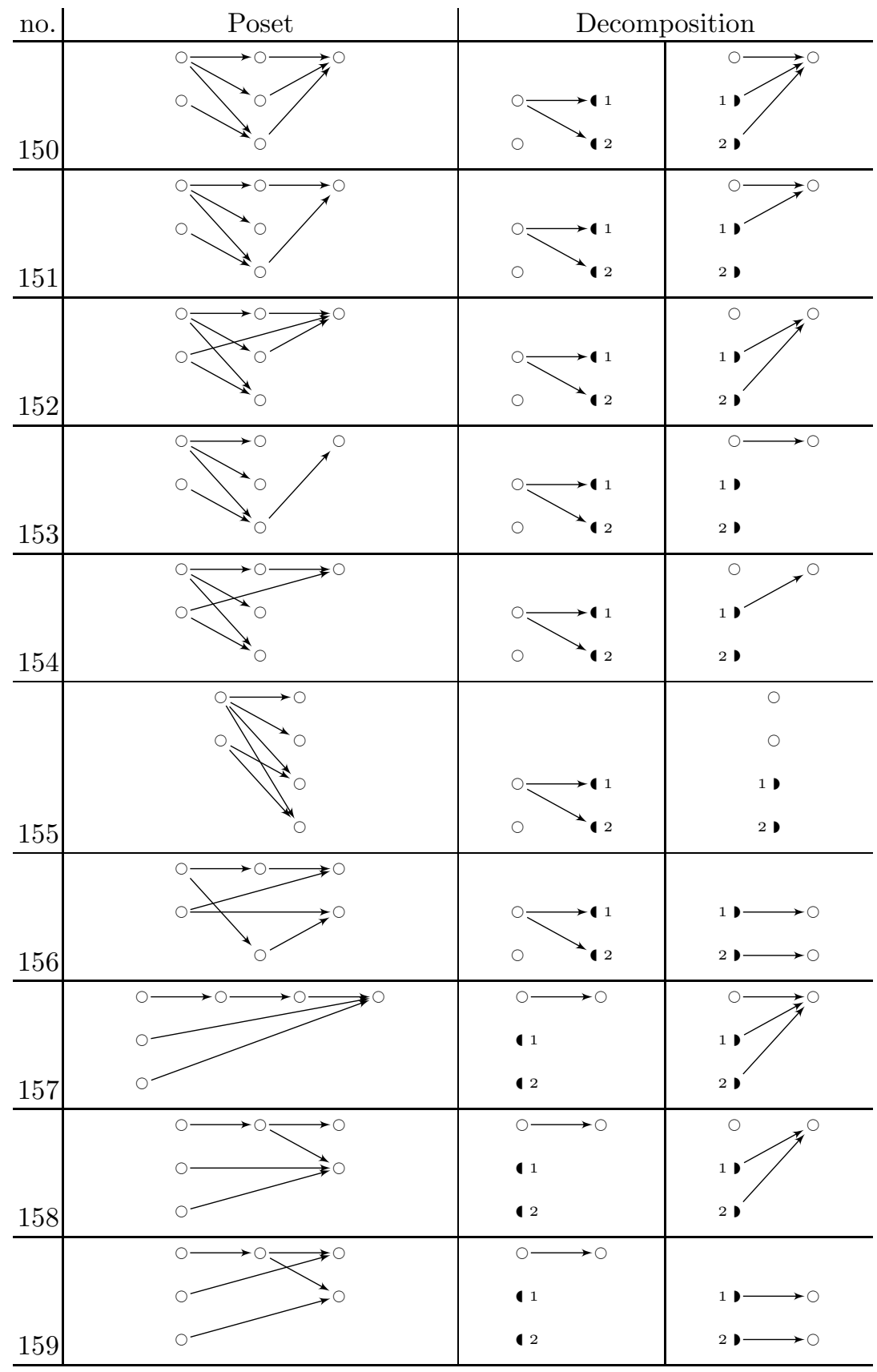


Table 4: Gluing-parallel decompositions of connected gp-posets on six points

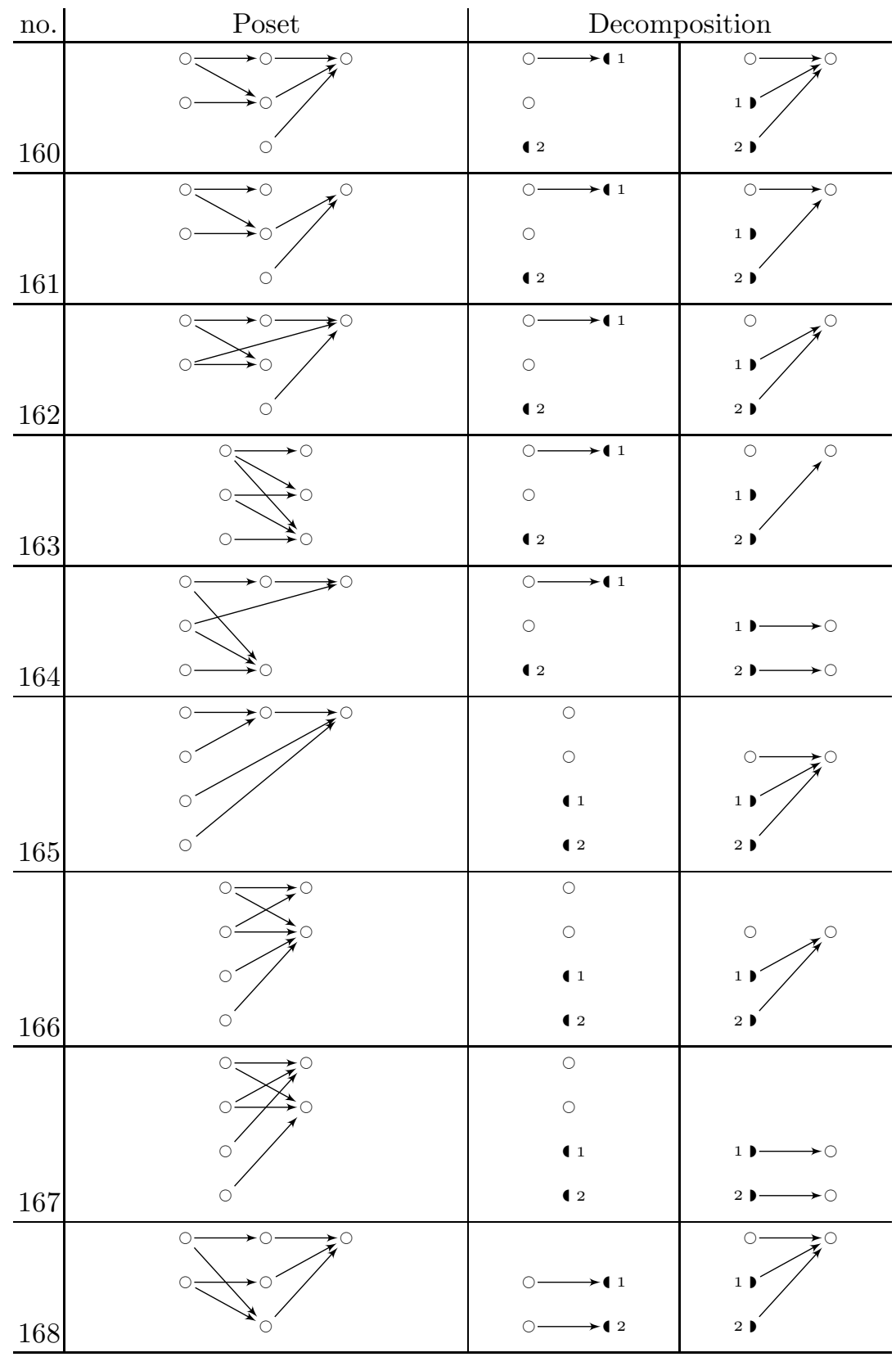


Table 4: Gluing-parallel decompositions of connected gp-posets on six points

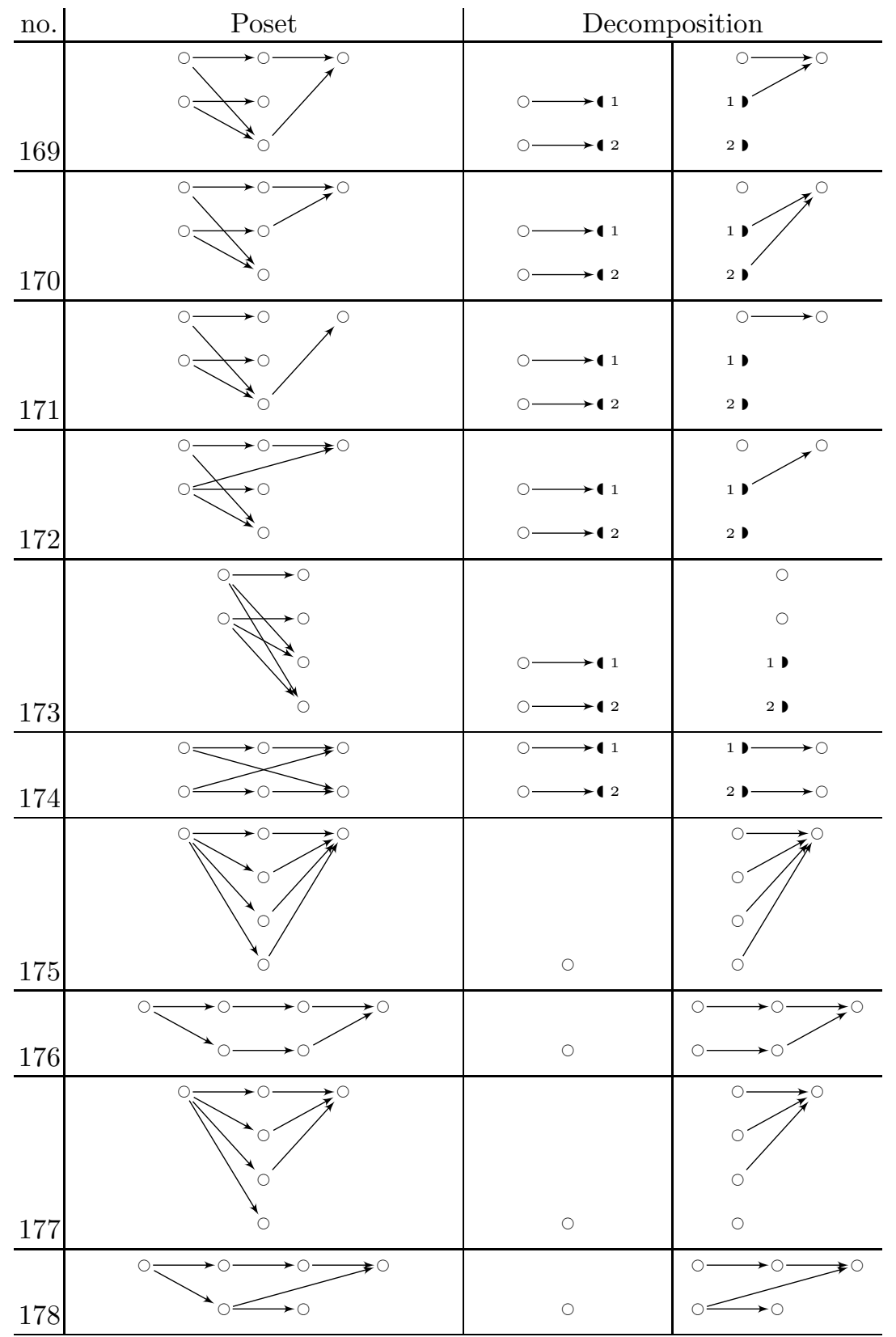


Table 4: Gluing-parallel decompositions of connected gp-posets on six points

\begin{tabular}{l|l|l|l}
\hline & \multicolumn{2}{|c}{ Decomposition } \\
\hline & & & \\
\hline
\end{tabular}


Table 4: Gluing-parallel decompositions of connected gp-posets on six points

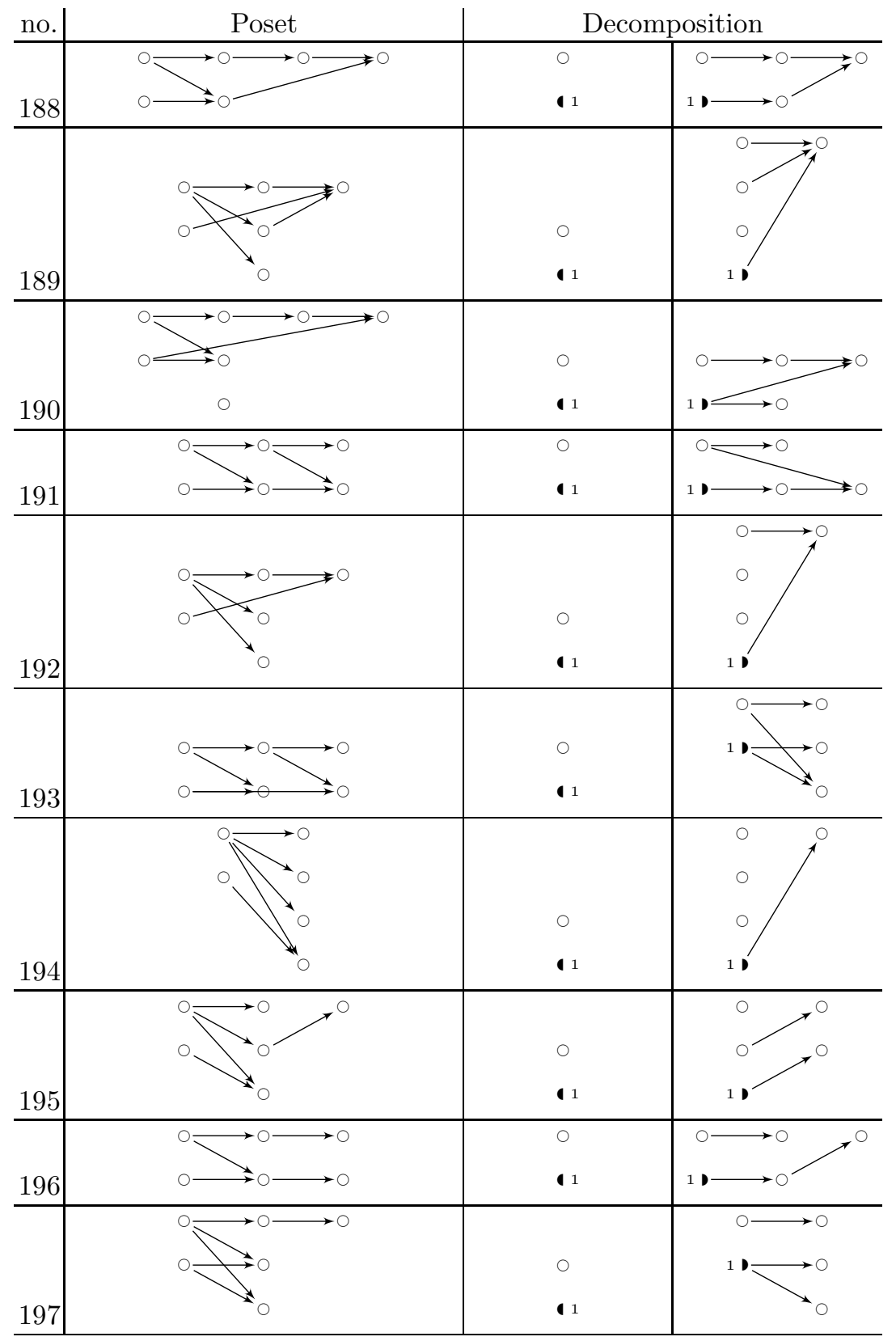


Table 4: Gluing-parallel decompositions of connected gp-posets on six points

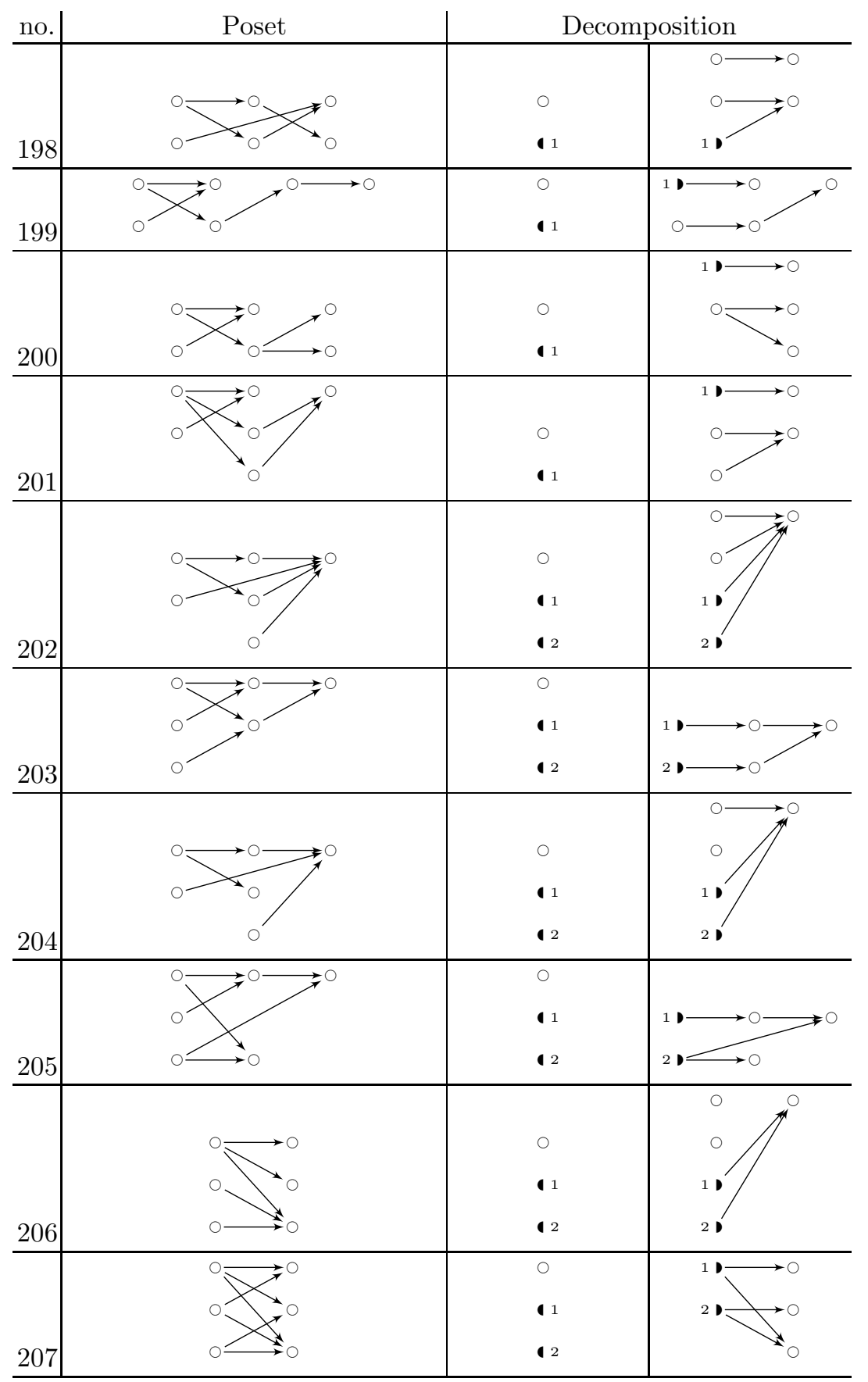


Table 4: Gluing-parallel decompositions of connected gp-posets on six points

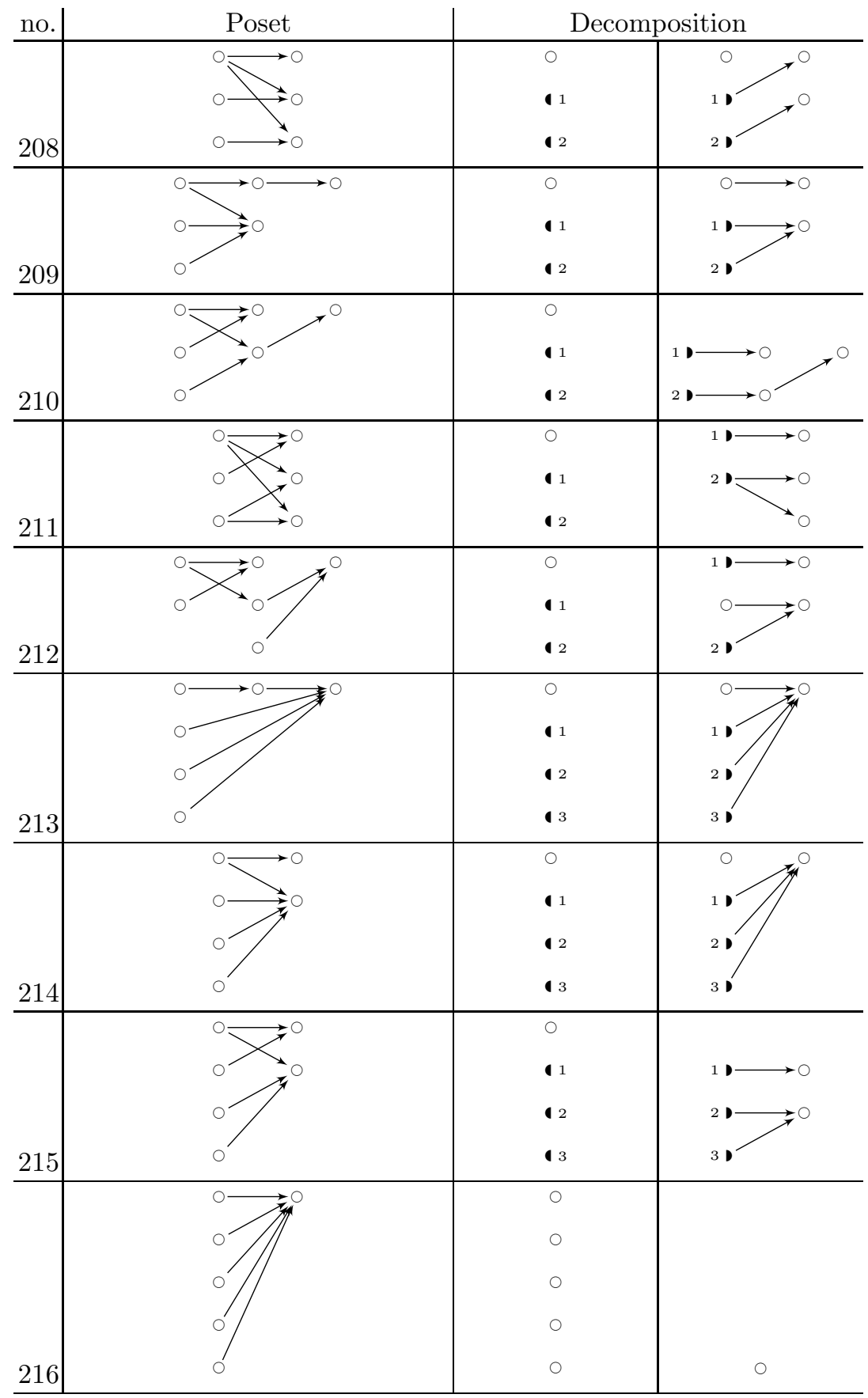


Table 4: Gluing-parallel decompositions of connected gp-posets on six points

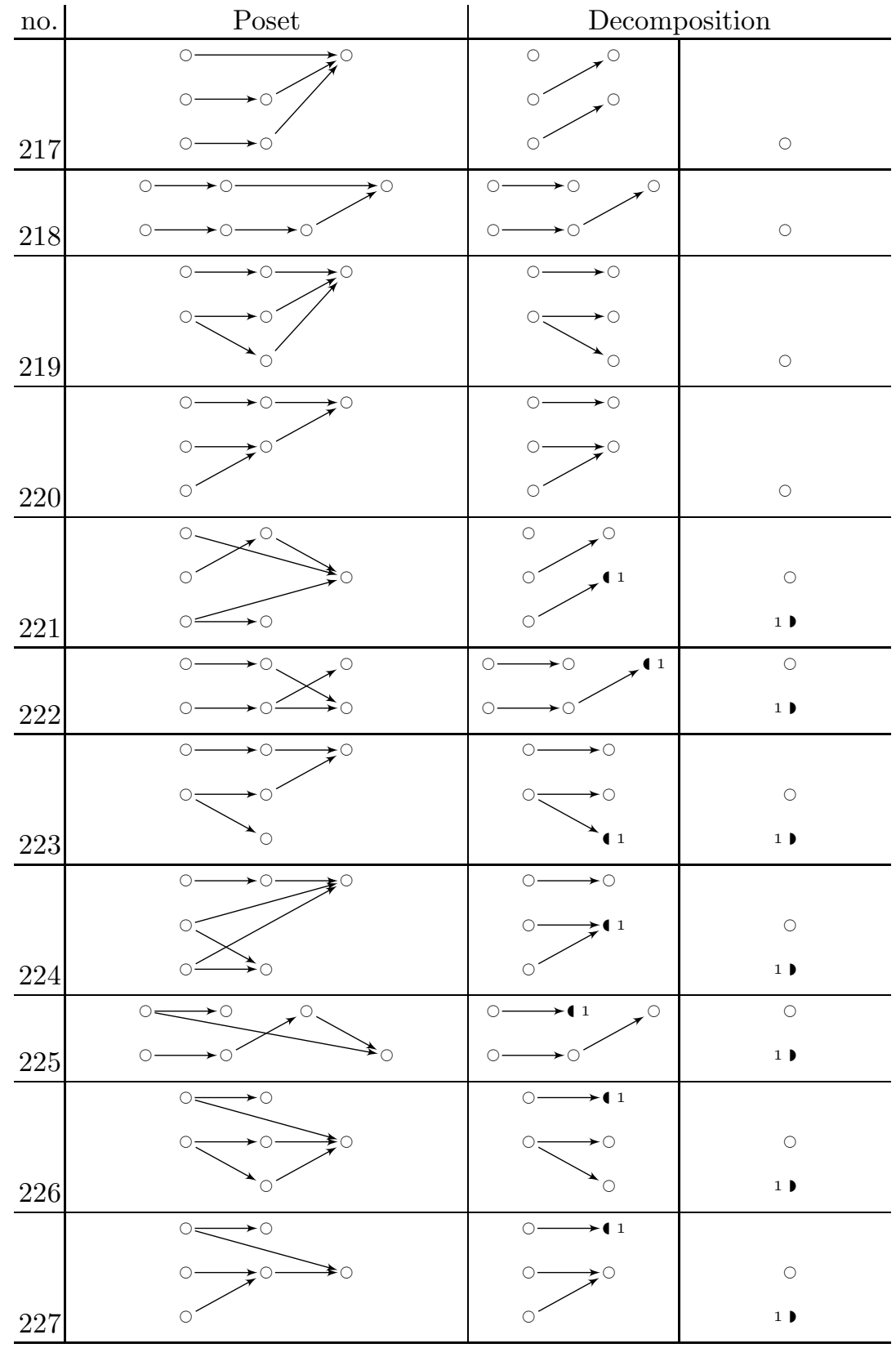


Table 4: Gluing-parallel decompositions of connected gp-posets on six points

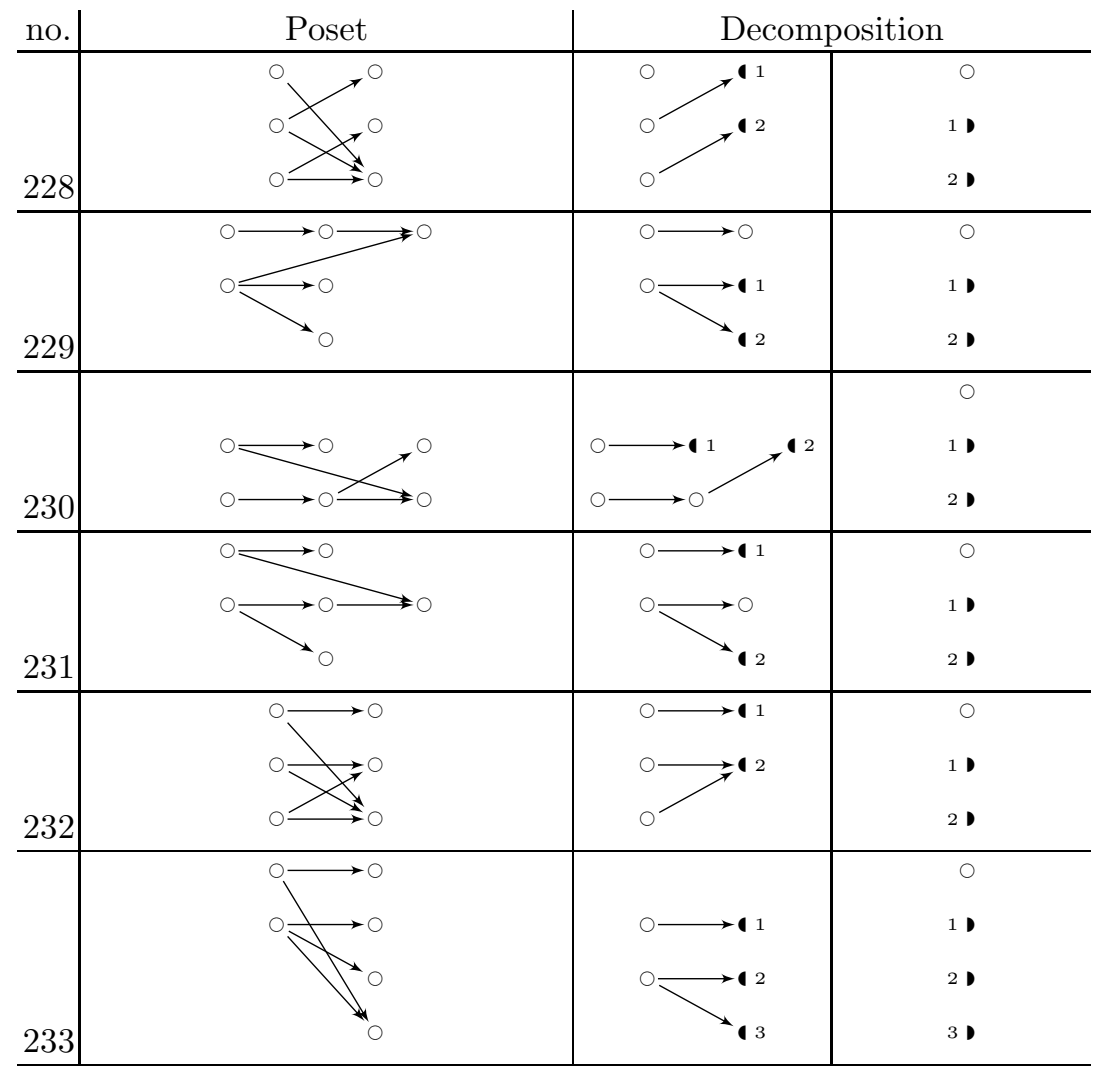

